\title{
VLA/JVLA monitoring of bright northern radio sources ${ }^{\star}$
}

\author{
N. Kurinsky ${ }^{1}$, A. Sajina ${ }^{1}$, B. Partridge ${ }^{2}$, S. Myers ${ }^{3}$, X. Chen ${ }^{4}$, and M. López-Caniego ${ }^{5}$ \\ 1 Tufts University, Medford, MA 02155, USA \\ e-mail: anna.sajina@tufts.edu \\ 2 Haverford College, Haverford, PA 19041, USA \\ ${ }^{3}$ National Radio Astronomy Observatory, Sorocco, NM 87891, USA \\ ${ }^{4}$ Infrared Processing and Analysis Center, California Institute of Technology, Pasadena, CA 91125, USA \\ 5 Instituto de Física de Cantabria (CSIC-Universidad de Cantabria), Avda. los Castros s/n, 39005 Santander, Spain
}

Received 20 June 2012 / Accepted 13 November 2012

\section{ABSTRACT}

\begin{abstract}
We report multiple epoch VLA/JVLA observations of 89 northern hemisphere sources, most with $37 \mathrm{GHz}$ flux density $>1 \mathrm{Jy}$, observed at $4.8,8.5,33.5$, and $43.3 \mathrm{GHz}$. The high frequency selection leads to a predominantly flat spectrum sample, with $85 \%$ of our sources being in the Planck Early Release Compact Source Catalog (ERCSC). These observations allow us to: 1) validate Planck's 30 and $44 \mathrm{GHz}$ flux density scale; 2) extend the radio spectral energy distributions of Planck sources to lower frequencies allowing for the full 5-857 GHz regime to be studied; and 3) characterize the variability of these sources. At $30 \mathrm{GHz}$ and $44 \mathrm{GHz}$, the JVLA and Planck flux densities agree to within $\sim 3 \%$. On timescales of less than two months the median variability of our sources is $2 \%$. On timescales of about a year the median variability increases to $14 \%$. Using the WMAP 7-year data, the $30 \mathrm{GHz}$ median variability on a $1-6$ years timescale is $16 \%$.
\end{abstract}

Key words. radio continuum: galaxies - galaxies: active - catalogs

\section{Introduction}

The Planck satellite (Planck Collaboration 2011a) is capable of all sky observation in nine bands ranging from 30 to $857 \mathrm{GHz}$. Planck's primary goal is to make sensitive observations of temperature and polarization anisotropies in the cosmic microwave background (CMB), but it also offers unprecedented frequency coverage of both Galactic and extragalactic sources, as many of Planck's bands lie in a frequency range at which accurate observations from the Earth's surface are very difficult due to atmospheric opacity. In addition, Planck has the ability to observe a given source at all frequencies essentially simultaneously, producing a wealth of spectral data much faster than ground based observatories can.

The first science product of Planck was the Early Release Compact Source Catalog (ERCSC; Planck Collaboration 2011b), which contains time-averaged flux densities of high reliability sources detected during the first year of Planck's operation, including 1.6 full sky surveys. The majority of ERCSC sources are brighter than $\sim 0.5-1.0 \mathrm{Jy}$, though minimum intensity is also dependent on the frequency of observation. In the low frequency bands considered in this paper, the ERCSC contains hundreds of extragalactic radio sources (Planck Collaboration $2011 b, c)$. These tend to be flat spectrum radio sources, predominantly blazars (Planck Collaboration 2011e; Giommi et al. 2011).

The photometric calibration of Planck is based on the largescale dipole introduced by the solar motion relative to the cosmic microwave background, an approach unlike the calibration used by ground based observatories. This dipole is very

^ Tables 3 and 4 are available in electronic form at http: //www . aanda.org well constrained, leaving a calibration uncertainty of $\sim 0.1 \%$. However, the absolute flux density calibration on very small scales (e.g. for point sources) also depends on a precise knowledge of the Planck beam at a given frequency, which is less well constrained. Our current understanding of the beam solid angle suggests an uncertainty on the order of $1 \%$. A cross-comparison of measured Planck and ground-based absolute flux density scales would therefore help either confirm that the adopted size of the Planck beam is within the expected uncertainty, or indicate that it may need further refinement. In addition, Planck provides a potential basis for future absolute flux density scales employed by ground-based facilities such as the Jansky Very Large Array (JVLA, formerly EVLA; Perley et al. 2011). To begin this process, an understanding of the current level of agreement between the two facilities is essential. Such a comparison, however, is complicated by the intrinsic variability of the sources that dominate the catalogs at the lowest Planck frequencies. This can be mitigated by observing as nearly simultaneously with Planck as possible, by using larger samples, and by better understanding of the intrinsic variability of the Planck sources.

In this paper, we present multiple-epoch VLA/JVLA observations of 89 sources in four spectral bands between 5 and $43 \mathrm{GHz}$, most of which are selected to be $>1 \mathrm{Jy}$ at $37 \mathrm{GHz}$, based on observations with the Metsähovi telescope (Hovatta et al. 2008, 2009). Preliminary results of this study were presented in Planck Collaboration (2011d). Based on 32 sources, a VLA-ERCSC flux density comparison found the median $30 \mathrm{GHz}$ ERCSC flux density to be $8 \pm 4 \%$ brighter than expected based on our VLA observations. Here we extend the previous analysis by including $\sim 3 \times$ more sources as well as using updated JVLA/VLA flux density standards. Unlike the case for our earlier paper, here we also have the advantage of multiple epoch observations of most sources that allow us to characterize the 
intrinsic variability of the sources over a range of timescales. Our VLA/JVLA observations were all scheduled to be nearly simultaneous with the survey by survey Planck observations of the same sources, although here we only make comparisons with the time averaged ERCSC data which are publicly available.

Aside from our program, there have been two other programs involving near-simultaneous observations of Planck-detected radio sources ${ }^{1}$. The first of these programs was the PACO project, conducted in the southern hemisphere (Massardi et al. 2011), which observed $20 \mathrm{GHz}$-selected sources using the Australia Telescope Compact Array (ATCA). Sources were observed essentially simultaneously at 5.5, 9, 18, 21, 33 and $39 \mathrm{GHz}$. The second of these programs was SiMPIE, which followed-up 250 $5 \mathrm{GHz}$-selected sources in the northern hemisphere using the single dish Medicina telescope at 5 and $8 \mathrm{GHz}$ as well as at $21 \mathrm{GHz}$, with observations made between Aug. 2010 and Aug. 2011 (Procopio et al. 2011). Compared to these surveys, our work covers a smaller number of sources (89); however, our predominantly $37 \mathrm{GHz}$ selection is most sensitive to the flat spectrum sources that dominate the Planck-detected radio sources. We use the JVLA whose $K a$ and $Q$-bands $(33.45$ and $43.22 \mathrm{GHz}$ respectively) closely match Planck's 28.46 and $44.10 \mathrm{GHz}$ bands. These characteristics make our sample particularly well suited to the flux density comparison presented in this paper. The SiMPIE study is the only one using a single dish radio telescope, and hence has the advantage of providing accurate flux densities for extended sources. While all of these programs have their strengths and weaknesses, the intrinsic variability of the Planck radio sources means that ultimately the more such ancillary data is collected close in time to Planck's observations of the same sources, the more value is added to the eventual Planck legacy point source catalog. Thanks to these three surveys, the vast majority of the brightest radio sources at $\sim 5-40 \mathrm{GHz}$ now have multiple epoch, near-simultaneous, ground-based observations. These observations extend the spectral energy distributions (SEDs) of the Planck detected blazars and flat spectrum radio galaxies to lower frequencies, and add data on the variability of these sources.

Our paper is organized as follows. In Sect. 2, we present the sample selection, observations, and data reduction. In Sect.3.1 we present the cross-matching of our sample with the ERCSC. In Sect. 3.2, we analyze the SED types of our sources. In Sect. 3.3, we characterize their variability. In this section, we also make use of data from the Wilkinson Microwave Anisotropy Probe (WMAP; Bennett et al. 2003) to provide a longer timescale for our variability analysis. Finally, in Sect. 3.4, we compare the Planck and JVLA flux densities at 30 and $44 \mathrm{GHz}$. The summary and conclusions are presented in Sect. 4.

\section{Data}

\subsection{VLA/JVLA sample}

Our main goal in selecting galaxies for our survey was to observe as many sources that would be bright enough at the $30-40 \mathrm{GHz}$ frequency range to have a high probability of being detected by the Planck Low Frequency Instrument (LFI; Mennella et al. 2011), which operates at frequencies of 30,44 , and $70 \mathrm{GHz}$. Our

\footnotetext{
1 Like our program, these are not true follow up studies of Planckdetected sources, which cannot be done simultaneously with Planck's observations, since Planck data reduction is time consuming. Instead, all three programs used their knowledge of Planck's scanning strategy combined with known samples of bright radio sources to produce samples likely to heavily overlap with detections made by Planck.
}

specific choice of VLA/JVLA observing targets was dictated by the goal of observing them as close to simultaneous with the Planck observations as possible.

The first results of our study presented in Planck Collaboration (2011d) included 32 sources chosen from the VLA calibrator list, where we specifically looked for 5 or $8 \mathrm{GHz}$ flux densities $>1 \mathrm{Jy}$. These published sources represent only the subset of the ones observed that were matched in the ERCSC at $30 \mathrm{GHz}$. In the present paper, we exclude the observations taken on July 24, 2009 since, for that day, we could not obtain primary flux calibrator measurements, but used a less reliable secondary calibrator. This leaves us with 23 of the sources in Planck Collaboration (2011d). We further include here data obtained on 16 sources in the same program on Nov. 15 and 18th, 2009, as well as on 11 more VLA sources that were not published in Planck Collaboration (2011d) because they did not have ERCSC counterparts.

In the present paper, we expand the sample with new JVLA data for 70 sources, which includes repeated observation of some of the above mentioned VLA sources. Our JVLA targets were predominantly selected from the Complete Northern $1 \mathrm{Jy}$ Sample, which includes all 104 sources above declination $-10^{\circ}$ whose average $37 \mathrm{GHz}$ flux density is $>1 \mathrm{Jy}$, as determined through long-term $37 \mathrm{GHz}$ monitoring by the Metsahovi telescope (e.g. Hovatta et al. 2008, 2009; Planck Collaboration $2011 \mathrm{e})^{2}$. In this paper, while the analysis focuses on the sources with ERCSC counterparts, we publish the data for all sources we observed near simultaneously with Planck for the sake of upcoming Planck catalogs which will reach lower flux densities than the ERCSC. We still exclude from the analysis three sources for which we measure $33 \mathrm{GHz}$ flux densities $<300 \mathrm{mJy}$ (these are cases where the wrong source was observed, or cases where the bulk of the flux is resolved out in our relatively high resolution imaging).

In summary, the sample we analyze includes 89 sources with a total of 159 separate observations. While the above makes it clear that the sample is neither homogeneous nor complete, 66 of our sources ( $75 \%$ of the total) are among the 104 in the Complete Northern 1 Jy Sample. These sources are therefore dominant, and largely determine the properties of our sample discussed in the rest of the paper. The spatial distribution of our sources can be seen in Fig. 1.

\subsection{Observations}

Our observations were made in two intervals of time, separated by approximately 6 months during which the NRAO array was down during the conversion from the VLA to the JVLA. In our earlier VLA observations, we used the $K$ band detectors, centered at $22.46 \mathrm{GHz}$, whereas after the conversion to the JVLA, we switched to the $K a$ band $(33.45 \mathrm{GHz})$. The $K a$ band is preferable to $K$ band, because it is closer to Planck's lowest frequency of $28.46 \mathrm{GHz}$, and also less affected by the atmospheric water vapor line at $22 \mathrm{GHz}$.

Observations were made in 23 observing blocks (Table 1$)^{3}$. In the case of the JVLA observations, these were typically $1 \mathrm{~h}$

\footnotetext{
2 However, in instances where there were insufficient numbers of $37 \mathrm{GHz}$-selected sources to fill the required $1 \mathrm{~h}$ scheduling blocks (see Sect. 2.2) sources selected as above were used to fill in the block.

3 For a few sources in our sample, we included earlier observations from Sajina et al. (2011) and, in the case of J0555+3948, earlier NRAO calibration observations (http://www.aoc.nrao.edu/ smyers/evlapolcal/polcal_master.html).
} 


\section{ERCSC F30GHz>1Jy \\ $\diamond$ Our VLA/EVLA follow-up ATCA follow-up (PACO)}

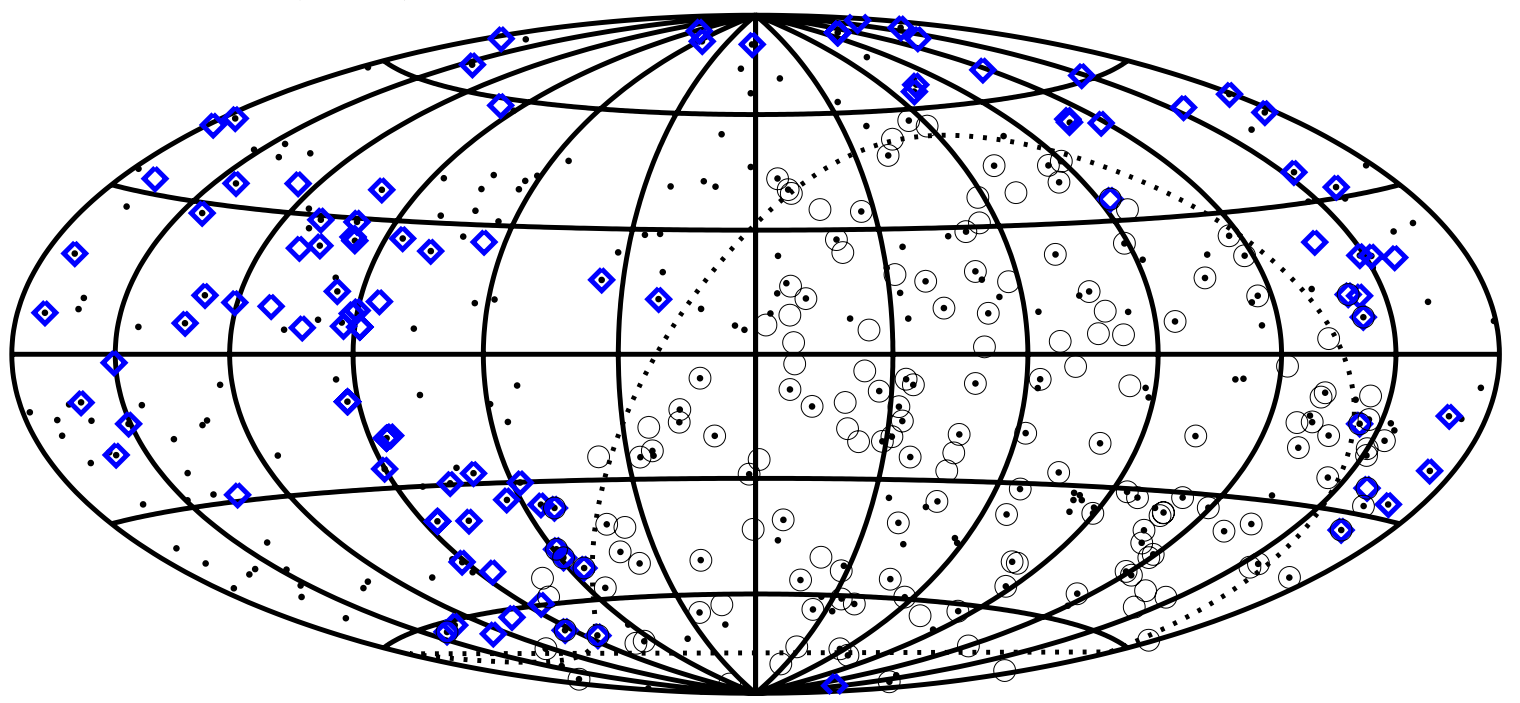

Fig. 1. Source map for the ERCSC and follow-up studies in Galactic coordinates. The dots mark positions of ERCSC $30 \mathrm{GHz}$ sources at high Galactic latitude $\left(|b|>5^{\circ}\right)$, with a $>1$ Jy cut applied, as at this level the sample is essentially complete. This is a total of 563 sources. Our study in the northern hemisphere includes 89 sources, the bulk of which are selected to have $S_{37 \mathrm{GHz}}>1 \mathrm{Jy}$. For comparison, we plot the $S_{20 \mathrm{GHz}}>1 \mathrm{Jy}$ sources from the AT20G survey (Murphy et al. 2010) in the southern hemisphere. All of these are part of the PACO project, where sources $>500 \mathrm{mJy}$ make up the PACO bright sample (Massardi et al. 2011). A dotted line separates the sky into the region covered by our study and that covered by the PACO project. Altogether, in the > 1 Jy regime, nearly $50 \%$ of the ERCSC $30 \mathrm{GHz}$ sources have ground-based, near-simultaneous follow-up.

Table 1. Scheduling blocks with bands observed, dates, and array configuration.

\begin{tabular}{lccc}
\hline \hline Scheduling block & Bands observed & Date & Configuration \\
\hline VLA 1 & $C, X, K, Q$ & Aug. 27, 2009 & $\mathrm{D}$ \\
VLA 2 & $C, X, K, Q$ & Oct. 22, 2009 & $\mathrm{D}$ \\
VLA 3 & $C, X, K, Q$ & Nov. 3, 2009 & $\mathrm{D}$ \\
VLA 4 & $C, X, K, Q$ & Nov. 15, 2009 & $\mathrm{D}$ \\
VLA 5 & $C, X, K, Q$ & Nov. 18, 2009 & $\mathrm{D}$ \\
VLA 6 & $C, X, K, Q$ & Dec. 11, 2009 & $\mathrm{D}$ \\
VLA 7 & $C, X, K, Q$ & Jan. 03, 2010 & $\mathrm{D}$ \\
JVLA 1 & $C, X, K a, Q$ & Jul. 2, 2010 & $\mathrm{D}$ \\
JVLA 2 & $C, X, K a, Q$ & Jul. 4, 2010 & $\mathrm{D}$ \\
JVLA 3 & $C, X, K a, Q$ & Jul. 9, 2010 & $\mathrm{D}$ \\
JVLA 4 & $C, X, K a, Q$ & Jul. 23, 2010 & $\mathrm{D}$ \\
JVLA 5 & $C, X, K a, Q$ & Aug. 3,2010 & $\mathrm{D}$ \\
JVLA 6 & $C, X, K a, Q$ & Sep. 7, 2010 & $\mathrm{D}$ \\
JVLA 7 & $C, X, K a, Q$ & Sep. 15, 2010 & $\mathrm{D}$ \\
JVLA 8 & $C, X, K a, Q$ & Sep. 20, 2010 & DnC \\
JVLA 9 & $C, X, K a, Q$ & Sep. 24, 2010 & DnC \\
JVLA 10 & $C, X, K a, Q$ & Oct. 18, 2010 & $\mathrm{C}$ \\
JVLA 11 & $C, X, K a, Q$ & Oct. 20, 2010 & $\mathrm{C}$ \\
JVLA 12 & $C, X, K a, Q$ & Nov. 8, 2010 & $\mathrm{C}$ \\
JVLA 13 & $C, X, K a, Q$ & Nov. 9, 2010 & $\mathrm{C}$ \\
JVLA 14 & $C, X, K a, Q$ & Nov. 20, 2010 & $\mathrm{C}$ \\
JVLA 15 & $C, X, K a, Q$ & Nov. 27, 2010 & $\mathrm{C}$ \\
JVLA 16 & $C, X, K a, Q$ & Nov. 30, 2010 & $\mathrm{C}$ \\
\hline
\end{tabular}

blocks. Each observing block included one of three standard calibrators (Table 2), as well as checks on array pointing, and 7-9 science targets. It is important to keep in mind that the bulk of these observations were done during very early stages of the JVLA's commissioning, when instrumental problems were to be anticipated. Given the brightness of our sources, even in the worst cases, the statistical error is below $0.5 \%$ (see Sect. 2.3 for details on observational errors and data flagging).

In all cases, the choice of specific targets to observe depended most strongly on the parts of the sky that the Planck satellite was scanning at the time of observation. With the queuebased system for JVLA scheduling, and given our higher demand for good weather, perfect simultaneity was not feasible. However, in the vast majority of cases, observations by Planck and the JVLA are separated by at most two weeks.

All of our observations were made with the VLA/JVLA array in its more compact configurations - D, C or DnC - where the angular resolution (the half power beam width) is $1-3^{\prime \prime}$ at $K$ band, $0.6-2^{\prime \prime}$ at $K a$ band and $0.5-1.5^{\prime \prime}$ at $Q$ band ${ }^{4}$ (22.46, 33.45, and $43.22 \mathrm{GHz}$ respectively). With the exception of J1230+1223 (i.e. M 87), our sources were unresolved even at the highest frequency in the highest resolution configuration, so we can generally compare flux density measurements made in different configurations. In comparison, the Planck beam widths, as adopted in the ERCSC are $32.65^{\prime}$ at $30 \mathrm{GHz}$ and $27.00^{\prime}$ at $44 \mathrm{GHz}$ (Planck Collaboration 2011b).

\subsection{Data reduction}

Data reduction of the JVLA observations was done using the CASA software package ${ }^{5}$. We first performed all the standard initial reduction steps, including correction for atmospheric opacity, antenna delay solutions, and bandpass corrections. In addition, $15-20 \%$ of the raw data had to be removed before further analysis due to errors related to receiver malfunction, temporarily de-comissioned antennae (due to the ongoing transition from

\footnotetext{
4 http://evlaguides.nrao.edu/index.php? title=0bservational_Status_Summary_-_Current\# Performance_of_the_JVLA

5 http://www. casa.nrao.edu
} 
Table 2. Flux densities of the primary JVLA calibrators.

\begin{tabular}{|c|c|c|c|c|c|c|c|c|c|c|}
\hline Calibrator & RA & Dec & $S_{C}(\mathrm{mJy})$ & $S_{X}(\mathrm{mJy})$ & $S_{K a}(\mathrm{mJy})$ & $S_{Q}(\mathrm{mJy})$ & $C_{C}$ & $C_{X}$ & $C_{K}$ & $C_{Q}$ \\
\hline 3С 048 & $01: 37: 41.2994$ & +33.09 .35 .1330 & 5437 & 3281 & 859 & 623 & 1.020 & 1.041 & 1.134 & 1.268 \\
\hline $3 \mathrm{C} 286$ & $13: 31: 08.2880$ & +30.30 .32 .9589 & 7394 & 5207 & 1893 & 1539 & 1.000 & 1.000 & 1.022 & 1.064 \\
\hline J0555+3948 & $05: 55: 30.8056$ & +39.48 .49 .1650 & 5339 & 4836 & 2681 & 2639 & - & - & - & - \\
\hline
\end{tabular}

Notes. The listed correction values $\left(C_{C}, C_{X}, C_{K}\right.$, and $\left.C_{Q}\right)$ given have been applied to the previously published VLA data (Planck Collaboration 2011d) and represent the ratio of the newer to the older flux density standards (see text for details).

VLA to JVLA), or time lapses in antenna slewing. Each source was amplitude and phase calibrated as well.

In each block, we included observations of one of three primary flux calibrators (Table 2), which provided the absolute flux density scale. We adopted the Perley-Butler 2010 absolute flux calibration standards. The VLA data originally presented in Planck Collaboration (2011d) were calibrated using the older Perley-Taylor 1999 standards, which we rescaled to the PerleyButler 2010 flux standards. Table 2 gives the adopted calibrator flux densities, as well as the correction factors applied to the earlier VLA data.

After calibration, an image was produced for each observation. Each target source was fitted with a two dimensional gaussian providing peak and integrated flux densities as well as their associated errors. We checked for extended sources by looking for discrepancies between peak and integrated fluxes and by examination of images. We found one extended source, J1230+1223 (i.e. M 87, 3C 274). In Table 3 we present the integrated flux densities and flux density errors for each source, observation, and band. The flux density errors listed represent the quadrature sum of the errors from the above fitting, which are dominated by the instrumental noise in the images, and calibration uncertainties which are determined by the standard deviation of the flux densities of 3C 48 and 3C 286 (the primary flux calibrators) at each frequency. At the $K a$ and $Q$ bands, the uncertainty introduced by the scatter in the measurements of the calibrators is estimated at $\sim 0.1-0.2 \%$.

\subsection{The early release compact source catalog}

In this paper, we obtain Planck flux densities for our sources from the Planck Early Release Compact Source Catalog (ERCSC; Planck Collaboration 2011b). The ERCSC contains high reliability (>90\% cumulative reliability) compact sources (both Galactic and extragalactic) based on Planck's first year of observations. In this paper, we adopt the standard flux density and flux density error estimates, as given in the FLUX and FLUX_ERR columns in the ERCSC catalogs. These are estimated through aperture photometry with a radius equal to the sky-averaged FWHM for the given band, with aperture corrections applied (Planck Collaboration 2011b). The ERCSC also includes flux density estimates based on fitting a 2D gaussian (GAUFLUX) as well as fitting the point-spread function PSFFLUX. The FLUX column is generally preferred since the GAUFLUX measurements are more sensitive to the presence of any underlying extended emission, while the PSFFLUX measure is affected by the fact that the exact shape of the Planck beam is not quite constant across the sky. In all cases, these flux densities are in fact averages of all data obtained for a given source by Planck observations at a given frequency. Since the ERCSC contains about 1.6 full sky surveys, the bulk of the sources have fluxes averaged over 2 or more time periods (with the sources closest to the ecliptic poles having the most frequent observations).
We note explicitly that the $44 \mathrm{GHz}$ Planck measurements were generally noisier than those at $30 \mathrm{GHz}$. In addition, as discussed in Planck Collaboration (2011d), the placement of the three $44 \mathrm{GHz}$ horns in the Planck focal plane ensured that there is an approximately one week separation in time between observations of a given source by one horn and the other two horns at that frequency. Thus variability is likely to play a larger role at $44 \mathrm{GHz}$ than at $30 \mathrm{GHz}$, where the horn separation is smaller.

\subsection{The WMAP 7-year data}

The WMAP data used in this paper comes from the WMAP Seven Year release (Jarosik et al. 2011; Gold et al. 2011). The 7-year catalog contains 471 sources which represent $5 \sigma$ peaks in the maps co-adding all data from the first 7 years of the survey in each of the 5 WMAP bands. The flux densities in this catalog are the mean flux densities of the sources across these 7 years. WMAP also produced single year maps, where (like the Planck observations) a given source has typically been observed more than once in the span of that year. Therefore even flux densities based on these single year maps are averaged over multiple observations. We obtain such yearly-averaged flux densities for the 471 sources in the 7-year catalog through the point source variability table 6 . Here we only make use of the WMAP $\mathrm{Ka}$ $(30.0 \mathrm{GHz})$ data.

\section{Results}

\subsection{Source matching}

We consider a given JVLA-ERCSC source pair a match if the distance between them is less than 0.75 FWHM of the Planck beam for a given band. As seen in Fig. 2, the positional offsets for our matches never exceed $\sim 6$ arcmin and are typically within $\sim 2$ arcmin. Given the scarcity of extragalactic radio sources at the $1 \mathrm{Jy}$ level, the chance of random association is negligible. However, in one case, the nominal Planck source is located in-between two of our sources (both listed as J1310+3220 in Table 3), and is the sum of the two. These sources are omitted from further analysis.

Of our 89 sources, we found 76 ERCSC matches at $30 \mathrm{GHz}$, and a subset of these (59) have ERCSC matches at $44 \mathrm{GHz}$ (listed in Table 4). The typically negative $30-44 \mathrm{GHz}$ spectral indices of our sources explain the lower number of matches at $44 \mathrm{GHz}$. This is also the result of the lower sensitivity of the Planck $44 \mathrm{GHz}$ band, as mentioned in Sect. 2.4.

\subsection{Sample characterization and SED types}

Figure 3 shows a diagnostic color-color plot, providing an overview of the SEDs of our sources. It is obvious that our

\footnotetext{
6 http://lambda.gfsc.nasa.gov/product/map/dr4/ ptsrc_variability_info.cfm
} 
N. Kurinsky et al.: VLA/JVLA monitoring of bright northern radio sources
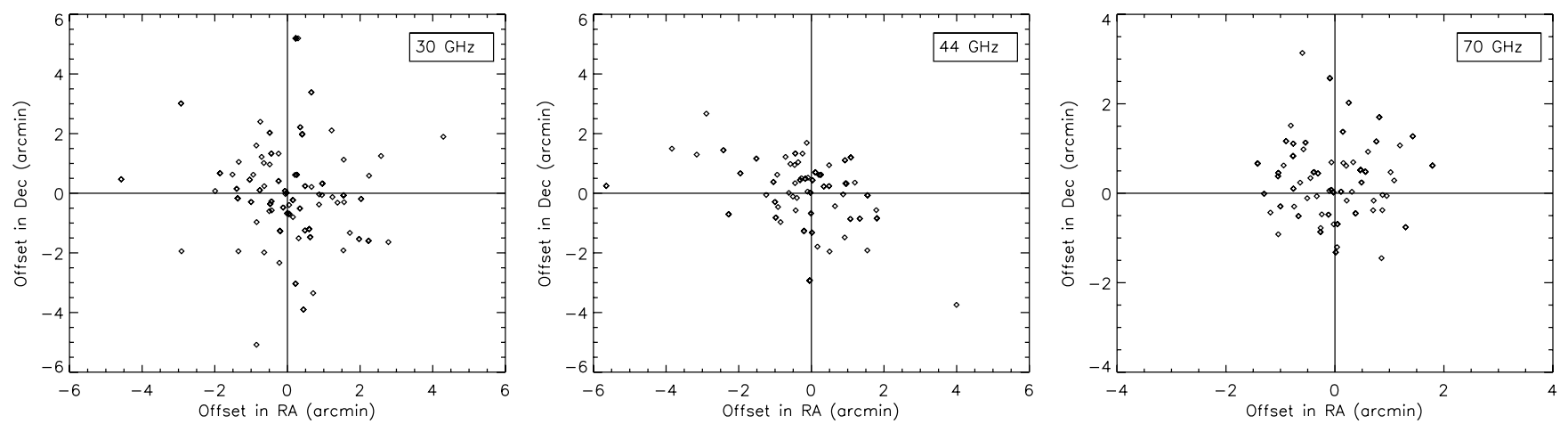

Fig. 2. Positional scatter between the Planck sources at 30, $44 \mathrm{GHz}$, and $70 \mathrm{GHz}$ and their JVLA matches. For the $70 \mathrm{GHz}$ comparison, the JVLA $K a$ positions are used.

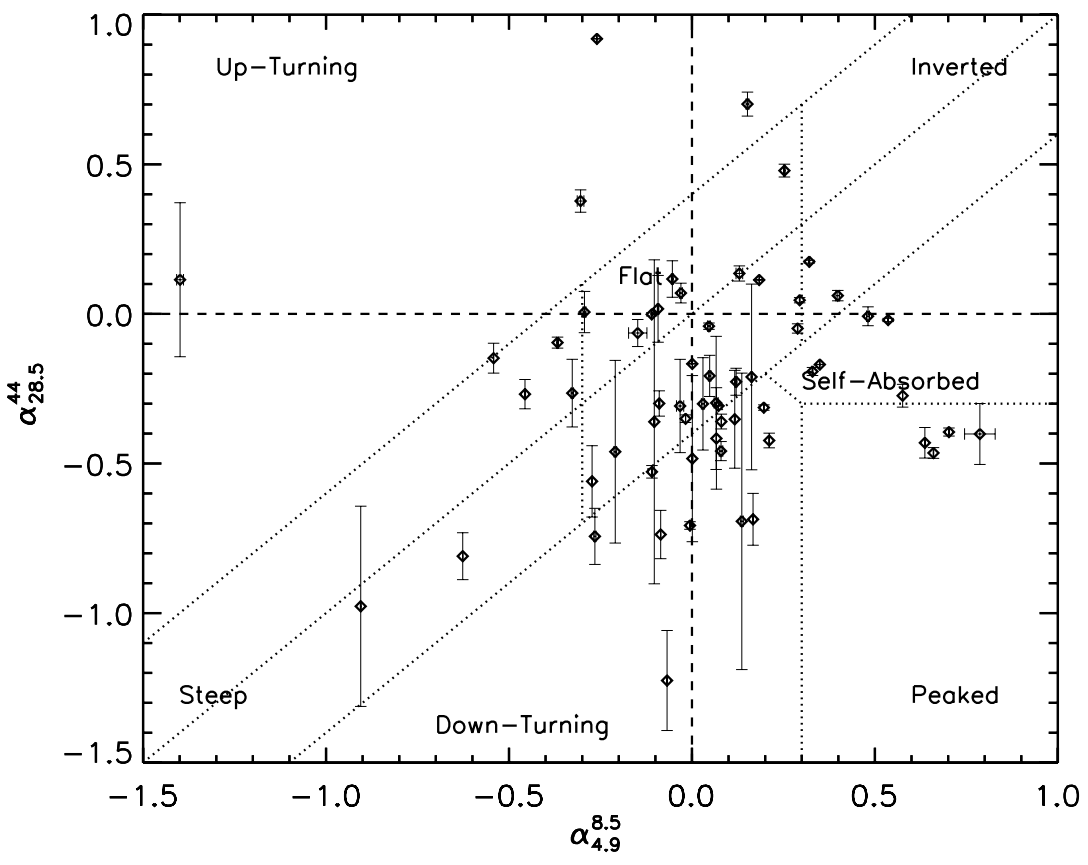

Fig. 3. 5-40 GHz radio source color-color diagnostic plot. Note that here we extrapolate both the VLA $K$-band and the JVLA $K a$-band observations to $28.46 \mathrm{GHz}$, the center frequency of the Planck $30 \mathrm{GHz}$ band. All observations for a given source are averaged, such that each data point represents a unique source. For ease of comparison, we adopt the Massardi et al. (2011) definitions of "flat", "steep", "up-turning", "inverted", "self-absorbed" and "peaked" spectra (as defined by the dotted regions).

Table 5. Relative frequency of different SED types, as defined in Fig. 3.

\begin{tabular}{lccc}
\hline \hline SED type & JVLA sample & PACO bright sample & PACO faint sample \\
\hline Flat & $42 \%$ & $10 \%$ & $5 \%$ \\
Steep & $10 \%$ & $4 \%$ & $13 \%$ \\
Down turning & $23 \%$ & $66 \%$ & $65 \%$ \\
Up turning & $7 \%$ & $0 \%$ & $0 \%$ \\
Inverted & $3 \%$ & $1 \%$ & $0 \%$ \\
Self absorbed & $8 \%$ & $5 \%$ & $5 \%$ \\
Peaked & $7 \%$ & $15 \%$ & $11 \%$ \\
\hline
\end{tabular}

Notes. Due to incomplete spectral coverage, this includes only 60 of our sources. The distribution of SED types for the PACO Bright and Faint samples are given for comparison (Massardi et al. 2011; Bonavera et al. 2011).

sample is biased toward flat-spectrum sources. In Table 5, we give the fractional distribution of each of the classes shown in Fig. 3, as well as the fractional composition of the PACO Bright and Faint samples (Massardi et al. 2011; Bonavera et al. 2011). We find that our sample has a much higher concentration of flat spectrum sources, compared with both PACO samples. This is unsurprising, given that the PACO selection is at $20 \mathrm{GHz}$, whereas our sample's primary selection is at $37 \mathrm{GHz}$. Naturally, the higher the frequency used to select a sample, the higher the fraction of flat spectrum relative to steep spectrum sources (e.g. Sajina et al. 2011). In addition, the PACO bright sample is based on $S_{20 \mathrm{GHz}}>0.5 \mathrm{Jy}$, whereas ours has a threshold of 


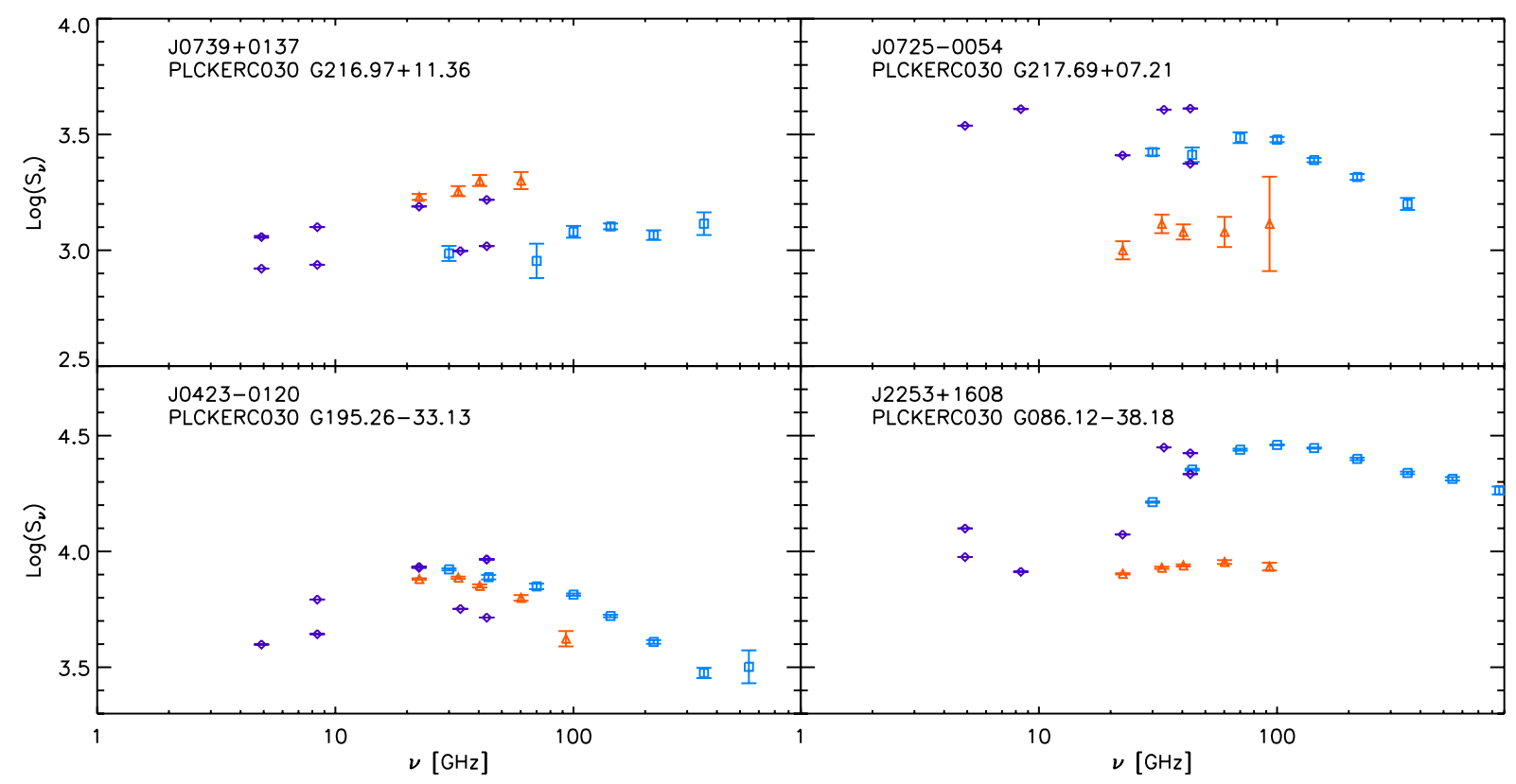

Fig. 4. The four most highly variable sources in our sample. The purple diamonds indicate VLA/JVLA data, orange triangles indicate the 7-year WMAP average flux densities of the sources, and light blue squares indicate the Planck ERCSC flux densities.

$S_{37 \mathrm{GHz}}>1 \mathrm{Jy}$ (for the $\sim 70 \%$ of the sample that are in the $1 \mathrm{Jy}$ Complete Northern Sample). Adopting a typical spectral index ${ }^{7}$ of -0.4 , our flux density limit translates to $1.3 \mathrm{Jy}$ at $20 \mathrm{GHz}$, brighter than the PACO bright sample limit.

As we demonstrate in Sect. 3.3, our sample shows significant variability. Four of the most highly variable sources are shown in Fig. 4, as an illustration. This variability means that a given source can be classified differently depending on when it is observed (both J0423-0120 and J2253+1608 clearly change the sign of their $\alpha_{28.5}^{44}$ from one observed epoch to the next). For simplicity, Fig. 3 is based on the average flux densities of our sources at each of the frequencies shown.

\subsection{Variability analysis}

The availability of multi-epoch observations allows us to study the variability of our sources, which is expected to be significant given that the bulk of our sample are flat spectrum sources, most likely blazars. For the variability analysis, we adopt the variability index formula from Sadler et al. (2006) which we reproduce in Eq. (1). In our implementation, we calculate the variability indices from pairs of observations, therefore $N=2$ in Eq. (1). The variability is assessed on the basis of the $K a(33 \mathrm{GHz})$ flux densities from multiple observations of the same source. The available $K(22 \mathrm{GHz})$ flux densities are included in the analysis, after being interpolated to the $K a$ central frequency using the $K-Q$ spectral indices.

$V_{\mathrm{rms}}=\frac{100}{\langle S\rangle} \sqrt{\frac{\sum\left(S_{i}-\langle S\rangle\right)^{2}-\sum \sigma_{i}^{2}}{N}}$.

Time separations between pairs of VLA/JVLA observations are in the range of 1-450 days. To allow us to look for trends in variability at different time scales, we divide this roughly into three intervals that are big enough to include a statistically significant number of sources. These are: "Interval 1" which covers 0-60 days with a median of 12 days, "Interval 2" which covers

\footnotetext{
7 Defined as $S \propto v^{\alpha}$.
}

60-260 days with a median of 168 days, and "Interval 3" which covers $290-430$ days with a median of 359 days. The number of sources included in each interval is $\sim 20$. Variability is computed for each pair of observations, which are sorted into the appropriate interval depending on their time separation. The vast majority of sources have only 2 separate observations, if any. In the rare cases where a source has two pairs of observations in the same bin, the two values are averaged. Figure 5 shows the distributions of the variability indices for the different time intervals along with their median values. Note that since the availability and level of multiple epoch observations varies strongly from source to source, this is only representative of the entire sample under the assumption that sources with more repeated observation are not biased toward being more or less variable than sources with fewer repeat observations. This is generally true, with one exception. The most frequently observed sources are the two primary flux calibrators: 3C 48 (J0137+3309) and 3C 289 (J1331+3030). These sources are used as primary JVLA calibrators precisely because of their very low variability. To remove this bias, we also show the median variabilities with these two sources removed. As expected, the median variabilities are now larger.

A total of 68 of our sources ( $76 \%$ of the sample) have been detected by WMAP based on the 7-year data release. This allows us to sample a fourth, longest term interval, "Interval 4", which includes time separation of 1-6 years. We make use of the yearly averages for our sources as found in the WMAP 7 year $K a(30 \mathrm{GHz})$ data. The time separation between any pair of these varies between 1 and 6 years with a median value of $\sim 2.5$ years. For the WMAP variability analysis, we apply Eq. (1) with $N=7$.

Figure 5 shows that the median variability of sources increases with timescale from $\sim 3 \%$ within a few weeks to $\sim 14 \%$ from year-to-year, with the largest variability $(\sim 16 \%)$ seen in the 1-7 year WMAP data. The fact that there is not a significant difference between our 1-year timescale variability and the 1-7 year WMAP variability estimate, is not surprising given that the median separation between the WMAP measurements is $\sim 2.5$ years (see above), which is not a large enough separation for us to detect a significant change in the variability. 
N. Kurinsky et al.: VLA/JVLA monitoring of bright northern radio sources

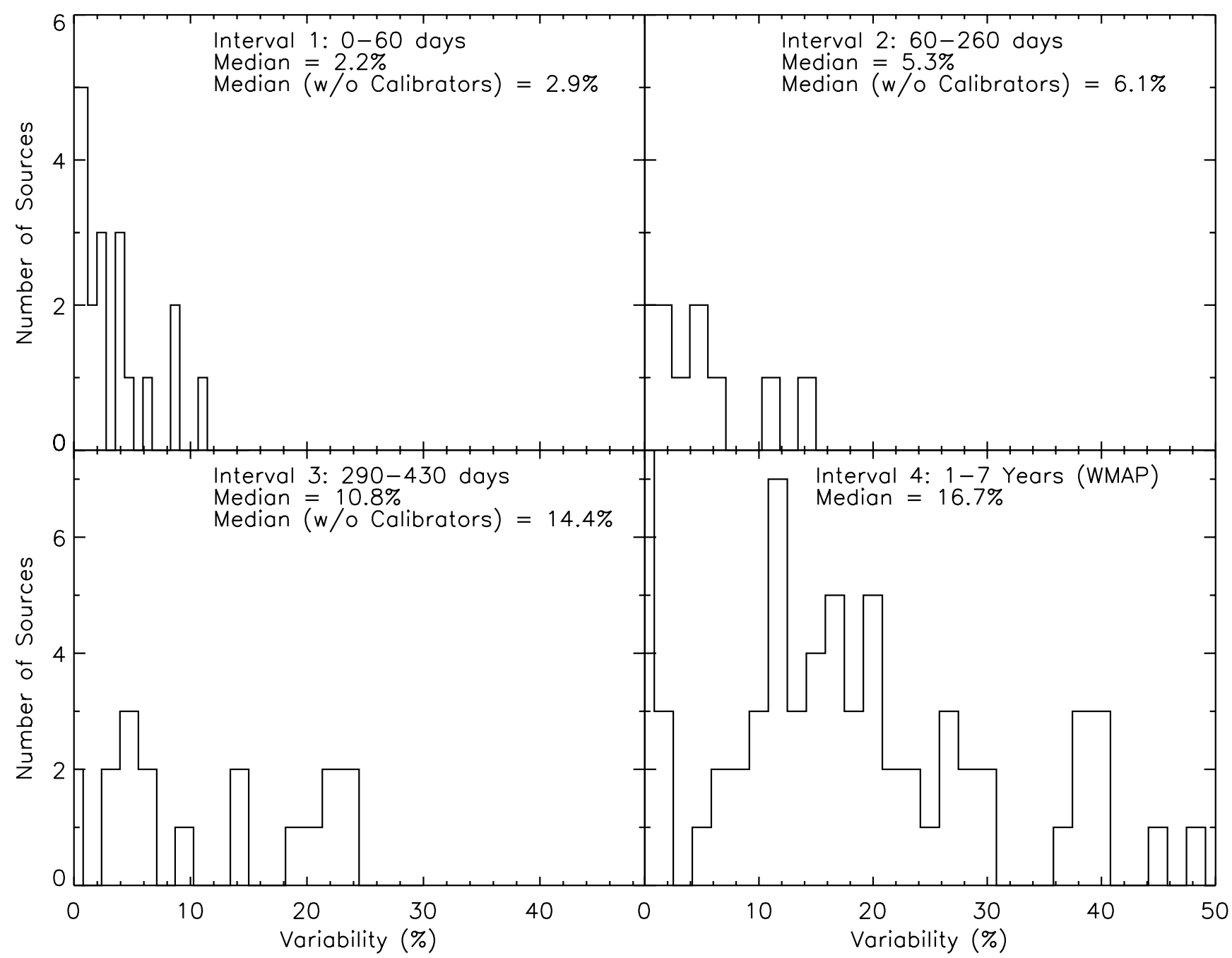

Fig. 5. Variability distribution on different timescales. The first three time intervals are based on our VLA/JVLA data alone. The last interval (1-7 years) is based on the yearly-averaged WMAP data (see text for details). All variability indices are estimated for the JVLA $\mathrm{Ka}$ band (33.5 GHz), or $33.0 \mathrm{GHz}$ for WMAP.

We also repeated the analysis for the $C$-band $(5 \mathrm{GHz})$ data to test how the variability of our sample changes with frequency as well as timescale. The results are summarized in Table 6. As expected, we found that the $C$-band variability is lower than at $\mathrm{Ka}$, especially on the 1 year timescale where it is $\sim 3 \%$ at $5 \mathrm{GHz}$ and $14 \%$ at $33 \mathrm{GHz}$.

The result that variability increases with frequency and timescale is consistent with the results found for the PACO bright sample (Massardi et al. 2011), although they only consider time intervals of 90,180 , and 270 days, whereas we also consider both shorter and longer timescales. They find that the $33 \mathrm{GHz}$ median variability of their sources increases from 6.7 to $10.6 \%$ between 90 and 270 days, although dipping slightly to $6.3 \%$ at 180 days. The $90-270$ day range is essentially our "Interval 2" in Fig. 5 where we find a median variability of $5.3 \%$ (after excluding the two calibrators). This is lower than the PACO-bright result, although, given the spread in Fig. 5, the two are marginally consistent. It is somewhat unexpected that PACO should find larger variabilities than we do, given that our sample includes more flat spectrum sources than the PACO-bright sample (see Table 5). The most likely explanation is that the variability index of a given source within a given time interval is based on the spread in flux densities around the mean flux density $(\langle S\rangle$ in Eq. (1)) for that particular time interval, whereas
Table 6. Median variability indices.

\begin{tabular}{lcccc}
\hline \hline Band & 0-60 days & $60-260$ days & $290-430$ days & $1-7$ years \\
\hline $5 \mathrm{GHz}$ & $1.0(1.1)$ & $4.0(4.3)$ & $3.0(3.3)$ & - \\
$33 \mathrm{GHz}$ & $2.2(2.9)$ & $4.0(5.3)$ & $10.8(14.4)$ & 15.6() \\
\hline
\end{tabular}

Notes. The values in parenthesis exclude the two calibrators $3 \mathrm{C} 48$ and 3C 286.

Massardi et al. (2011) take the $\langle S\rangle$ to be the mean flux density based on all available observations of a given source. Given that variability increases with timescale, the quoted PACO variabilities are therefore likely to be biased high relative to ours. We choose to do our analysis in the manner described above, since: 1) the uneven time coverage of our data does not allow us to adopt equivalent "mean flux densities" for all sources; and 2) given the result that variability increases with time, adopting the mean flux density for a larger timescale than considered biases the estimated variability indices for a given interval. We refer the reader to Chen et al. (2012) for a much more detailed analysis of of the variability of the ERCSC sources based on WMAP data, including a discussion on the limitations of the commonly used variability index formula which we adopt here. 


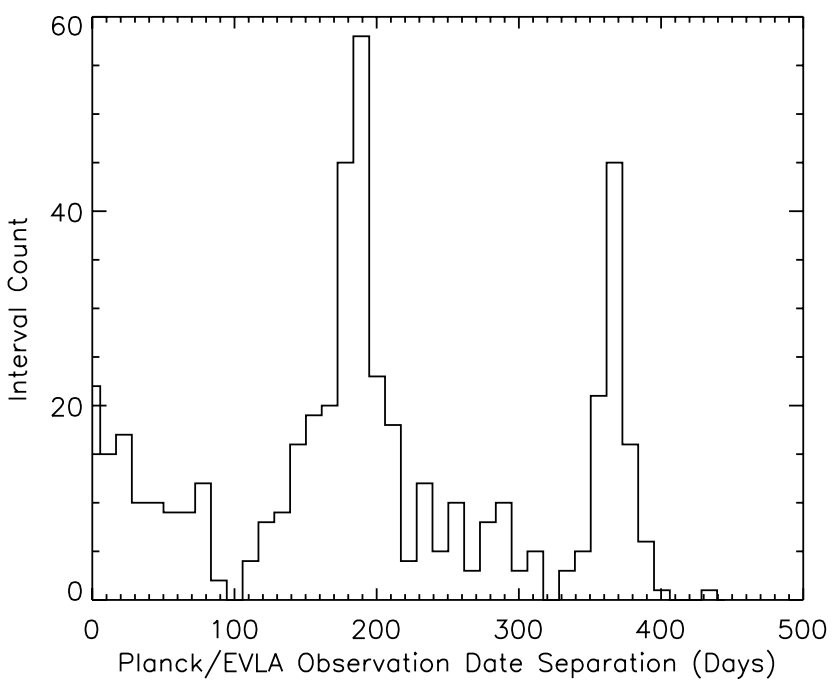

Fig. 6. Time intervals between our JVLA observations of a given source and the associated observations in the ERCSC. It is important to note that the bulk of our JVLA observations were actually taken after the end of period included in the ERCSC, but were timed so that in the majority of cases they were within a few weeks of the later Planck observations of the same source.

Lastly, Fig. 6 shows the distribution of time lags between ERCSC observations and each VLA/JVLA observation for the sources in our sample. The two most prominent peaks correspond to approximately six months and one year after the Planck observations included in the ERCSC. These arise because a typical source is revisited by Planck every six months. Our earlier VLA observations correspond to the time of Planck's observations included in the ERCSC. Hence our later JVLA observations, done close in time to Planck's observations of the same source, occur typically 6 months and then a year after the ERCSC observations. Given the above analysis, we expect the observed variability in comparing the ERCSC and VLA/JVLA observations to be $\sim 6-14 \%$, as shown in Fig. 5 and Table 6.

\subsection{JVLA-ERCSC flux density comparison}

Here we compare the Planck ERCSC and JVLA flux density scales at $30 \mathrm{GHz}$ and $44 \mathrm{GHz}$. Although our observations were scheduled to be close in time to Planck observations of the same sources, the comparison here is done against the public ERCSC and our survey extends about a year past the end of the period included in the ERCSC (see Fig. 6). In addition, the ERCSC itself includes data averaged over 1.6 sky surveys. Since we lack time ordered data from Planck for the time of the JVLA followup, and given the variability of our sources (see Sect.3.3), individual source flux comparison is not meaningful. However, a statistical comparison can still be done. To do so, we extrapolate the $K a(K)$ ground-based flux densities to the Planck $30 \mathrm{GHz}$ and $44 \mathrm{GHz}$ bands (centered at $28.46 \mathrm{GHz}$ and $44.1 \mathrm{GHz}$ respectively). These extrapolations are done by assuming a power law between the $K a$ (or $K$ ) to $Q$ bands with a spectral index as measured for each VLA/JVLA observation of a given source. We also apply color corrections to the Planck ERCSC flux densities. The multiplicative color corrections from the ERCSC Explanatory Supplement ${ }^{8}$ are also based on the $K a-Q$ spectral index, but as measured from the ERCSC data, whenever both

\footnotetext{
8 http://irsa.ipac.caltech.edu/data/Planck/ explanatory_supplement.pdf
}

30 and $44 \mathrm{GHz}$ matches are found, or from the VLA/JVLA data otherwise.

As stated in Sect. 3.1, 76 of our sources have ERCSC $30 \mathrm{GHz}$ matches and 59 have $44 \mathrm{GHz}$ matches. For the flux density comparison, we exclude M 87 which is extended to the JVLA, as well as the two J1310+3220 sources which are blended in the ERCSC. Next, we exclude 8 sources whose WMAP variability index is $>30 \%$, as well as $\mathrm{J} 0359+5057$, which is very close to the Galactic plane $\left(b=-1.6^{\circ}\right)$ making its photometry less reliable. All other sources in the sample are sufficiently far from the Galactic plane (see Fig. 1) for Galactic emission to be negligible. Finally, we look for remaining outliers, i.e. sources whose ERCSC and JVLA flux densities are more than a factor of 2 discrepant. There are two such cases: J0418+3801 and $\mathrm{J} 1751+0939$. A literature search revealed that J0418+3801 (3C 111) is known to be a double lobed radio galaxy (Leahy et al. 1997). Our measurements are consistent with earlier VLA measurements of the core flux, but Planck's much larger beam will respond to the extended structure resolved out in our interferometric observations. As for J1751+0939, our JVLA flux density measurements are below earlier data from both the VLA and WMAP. The source is variable, however, with a WMAP variability index just below our $30 \%$ cutoff mentioned above; therefore, it is possible that our observations were made at a time of particularly weak radio emission. Since our estimates at the four frequency bands are all consistent among themselves, it is less likely that this discrepancy is due to a mistake. We exclude $\mathrm{J} 0418+3801$ and $\mathrm{J} 1751+0939$ from the flux density comparison. This leaves us with a clean sample for the flux density comparison including 63 sources at $30 \mathrm{GHz}$ and 49 sources at $44 \mathrm{GHz}$.

Figure 7 shows the 30 and $44 \mathrm{GHz}$ JVLA-to-ERCSC flux density comparisons. Whenever applicable, we use the average flux densities based on all our VLA/JVLA observations of the same source. We next want to quantify the level of agreement between the JVLA and Planck ERCSC flux density scales. To minimize the possible effects of any remaining outliers, we compute the robust mean of each ratio, where $3 \sigma$ outliers are excluded. At both 30 and $44 \mathrm{GHz}$, this removes just 2 sources. At $30 \mathrm{GHz}$, the robust mean of the Planck-to-JVLA ratio is $1.006 \pm 0.026$, while at $44 \mathrm{GHz}$ it is $1.053 \pm 0.040$. In both cases, these values are clearly affected by Eddington bias (Eddington 1913) at the lower flux densities. To minimize this bias, we also compute the robust mean and median values for the sources where the extrapolated JVLA flux density is $>1.5 \mathrm{Jy}$. With this flux density cut, no sources were excluded in the calculation of the robust mean. We find the robust mean(median) at $30 \mathrm{GHz}$ is now $1.008(0.977)$ and at $44 \mathrm{GHz}$ it is $0.983(0.993)$.

The above analysis suggests that the Planck ERCSC and (J)VLA flux densities are consistent with each other to within $\sim 2-3 \%$ at both $30 \mathrm{GHz}$ and $44 \mathrm{GHz}$. This level of statistical agreement does not apply to individual sources, whose flux density uncertainties can be much larger. The best example is the primary (J)VLA flux density calibrator 3C $286(\mathrm{~J} 1331+3030)$, a well studied source with very low intrinsic variability. Compared to expectations from the JVLA, 3C 286 has a nearly $20 \%$ lower $30 \mathrm{GHz}$ flux density in the ERCSC ${ }^{9}$. Only a $30 \%$ accuracy in the photometry is required for inclusion in the ERCSC, whose emphasis is on the reliability of the sources.

Figure 7 shows significant scatter, which is clearly larger than the nominal ERCSC flux density errors, which in turn are larger than the JVLA errors. Given the median variabilities on a

\footnotetext{
9 Although it is in agreement with expectations if the ERCSC's GAUFLUX value is used.
} 
N. Kurinsky et al.: VLA/JVLA monitoring of bright northern radio sources
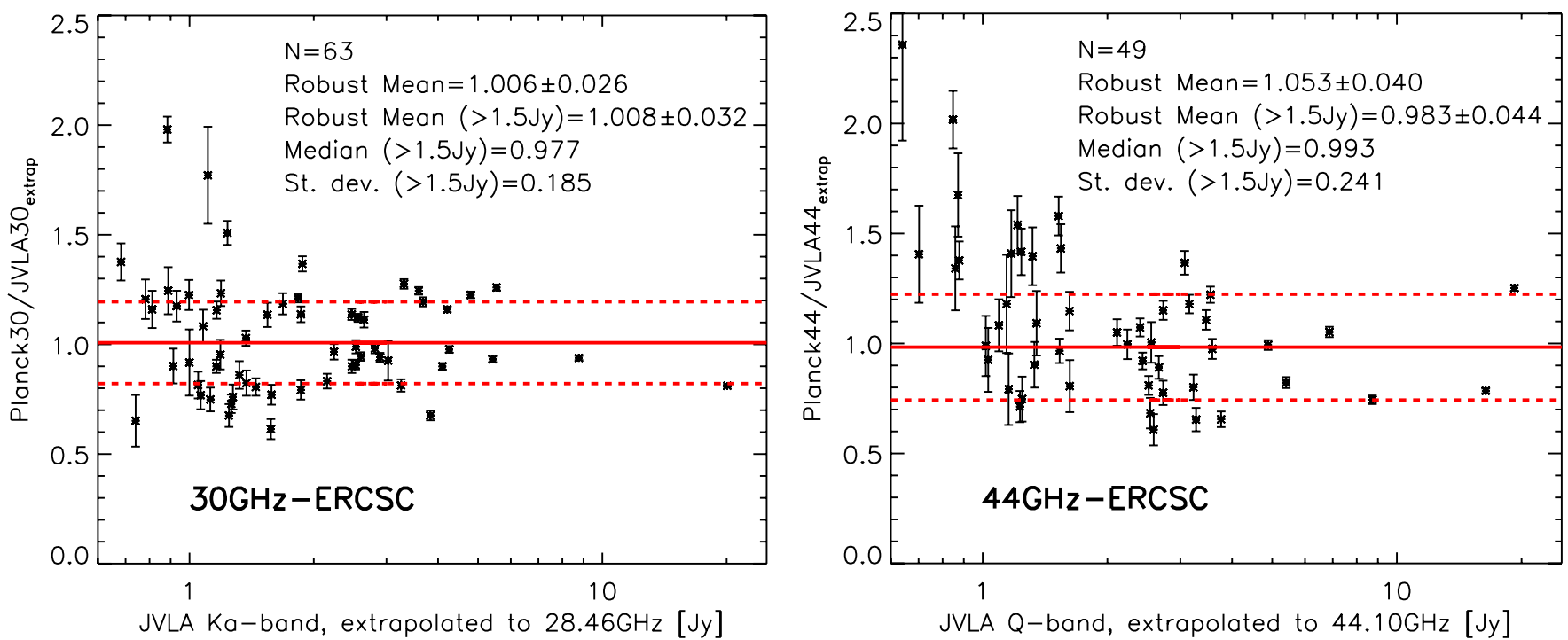

Fig. 7. Ratio of Planck-to-JVLA flux densities vs. JVLA flux densities at 30 (left-hand panel) and $44 \mathrm{GHz}$ (right-hand panel). The Planck flux densities used are from the FLUX column of the ERCSC. The JVLA flux densities are extrapolated to the corresponding Planck central frequencies using the 33-43 GHz spectral index measured from the JVLA data. Some sources are excluded from this analysis, due to being extended, confused, or highly variable (see text for details). When multiple observations for a given source are available, these are averaged. Each panel shows the number of sources included, as well as the robust mean, median and standard deviations of the Planck-to-JVLA flux density ratios. To mitigate the effects of Eddington bias, we focus on the $>1.5 \mathrm{Jy}$ sources. The solid red lines show the median ratios for these sources, while the dashed red lines show the $+/-1$ standard deviation range.

$\sim 6$ months to a year timescale (see Fig. 5), we predict the scatter to be in the range $6-14 \%$. This is lower than the measured standard deviation of $19 \%$, which may be due to underestimated variability indices as well as additional flux density uncertainties. The nominal ERCSC flux density uncertainties are $\sim 3 \%$, but including systematics they would have to be $\sim 15 \%$ to account for the observed scatter. This is within the discrepancy seen between the different flux density estimates (even within the ERCSC) for the essentially non-variable 3C 286.

As noted in Sect. 1, the accuracy of Planck's calibration on small angular scales, including point sources, is determined by how well the Planck beam is known - in essence, the flux density scales as $\theta^{2}$, where $\theta$ is the FWHM of the beam. Here we use the Planck flux density values as tabulated in the ERCSC, where the FWHM of the $30 \mathrm{GHz}$ beam is $32.65 \mathrm{arcmin}$, and of the $44 \mathrm{GHz}$ beam it is 27.00 arcmin. Work is ongoing to further refine our understanding of the Planck beams. Our results indicate that the Planck beams are unlikely to be revised by more than $\sim 2 \%$, if we assume the JVLA flux density scale is completely accurate. If they are revised more significantly over time, this may indicate that it is the JVLA flux density scale that needs to be revised. Future work (Perley et al., in prep.) based on time ordered Planck data (which also include the observations presented here) will help further constrain the level of agreement between the two instruments.

\section{Summary and conclusions}

1. We present new 5,8,33, and $43 \mathrm{GHz}$ observations for a sample of 89 bright ( $>70 \%$ selected to have $S_{37 \mathrm{GHz}}>1 \mathrm{Jy}$ ) northern radio galaxies. Our observations were scheduled to be within a few weeks of the Planck coverage of these intrinsically variable sources, allowing for a range of studies including SED and variability analysis that shed light on the nature of the Planck detected radio sources. This study is complementary to similar work done in the southern hemisphere by the PACO project (e.g. Massardi et al. 2011; Bonavera et al. 2011) and in the northern hemisphere by the SiMPIE project (Procopio et al. 2011). Our study has the highest fraction of sources in the ERCSC (85\%), thanks to its selection which favors higher flux density and flatter spectrum sources than PACO or SiMPLE.

2. From standard color-color diagnostic plots, we find that roughly $1 / 2$ of our sources are flat-spectrum, $1 / 5$ are steep spectrum, 1/5 are down-turning, and the remainder are up-turning, inverted, or self-absorbed. Examination of the $5-857 \mathrm{GHz}$ SEDs shows that classification on the basis of two spectral indices is over simplistic and varies strongly depending on the frequency examined.

3. Multiple VLA/JVLA observations for nearly half the sources, along with archival WMAP data, allow us to assess the level of variability in bright radio galaxies on a range of timescales from several weeks to several years. We find that the median variability increases with timescale, from $3 \%$ (about 2 weeks) to $16 \%$ (about 2.5 years).

4. We compare the flux density scales for Planck's two lowestfrequency LFI bands and the JVLA's $K a$ and $Q$ bands. The agreement between the two instruments is within $\sim 2-3 \%$ at $30 \mathrm{GHz}$ and $44 \mathrm{GHz}$. This is a significant improvement over previously reported results (Planck Collaboration 2011d), thanks to a larger sample, an improved JVLA flux density calibration, and better removal of the intrinsically most variable sources.

Acknowledgements. We are grateful to the anonymous referee for a careful reading of the paper and many useful suggestions which have improved the quality and presentation of the paper. We are particularly grateful to the Planck Collaboration for providing the public Early Release Compact Source Catalog (ERCSC). A description of the Planck Collaboration and a list of its members, including the technical or scientific activities in which they have been involved, can be found at http://www.sciops.esa.int/index.php? project=planck\&page=Planck_Collaboration. This paper makes use of observations obtained at the Jansky Very Large Array (JVLA) which is an instrument of the National Radio Astronomy Observatory (NRAO). The NRAO is a facility of the National Science Foundation operated under cooperative agreement by Associated Universities, Inc. We are especially grateful to Leon Tavares for providing source lists of sources, drawn from the Complete Northern $1 \mathrm{Jy}$ sample, to be observed by Planck in a given week. We are also grateful to 
Charles Lawrence and Joaquin Gonzales-Nuevo for helpful discussions and reviews of the manuscript before submission. This work was funded through a subcontract to Tufts University, and another to Haverford College from NASA/JPL in support of Planck related activities. MLC acknowledges financial support from the Spanish MINECO projects AYA2010-21766-C03-01 and CSD201000064. The development of Planck has been supported by: ESA; CNES and CNRS/INSU-IN2P3-INP (France); ASI, CNR, and INAF (Italy); NASA and DoE (USA); STFC and UKSA (UK); CSIC, MICINN and JA (Spain); Tekes, AoF and CSC (Finland); DLR and MPG (Germany); CSA (Canada); DTU Space (Denmark); SER/SSO (Switzerland); RCN (Norway); SFI (Ireland); FCT/MCTES (Portugal); and The development of Planck has been supported by: ESA; CNES and CNRS/INSU-IN2P3-INP (France); ASI, CNR, and INAF (Italy); NASA and DoE (USA); STFC and UKSA (UK); CSIC, MICINN and JA (Spain); Tekes, AoF and CSC (Finland); DLR and MPG (Germany); CSA (Canada); DTU Space (Denmark); SER/SSO (Switzerland); RCN (Norway); SFI (Ireland); FCT/MCTES (Portugal); and PRACE (EU).

\section{References}

Bennett, C. L., Halpern, M., Hinshaw, G., et al. 2003, ApJS, 148, 1 Bonavera, L., Bonaldi, A., Massardi, M., et al. 2011, MNRAS, 416, 559
Eddington, A. S. 1913, MNRAS, 73, 359

Giommi, P., Polenta, G., Lahteenmaki, A., et al. 2012, A\&A, 541, A160

Gold, B., Odegard, N., Weiland, J. L., et al. 2011, VizieR Online Data Catalog, 219, 20015

Hovatta, T., Nieppola, E., Tornikoski, M., et al. 2008, A\&A, 485, 51

Hovatta, T., Valtaoja, E., Tornikoski, M., \& Lähteenmäki, A. 2009, A\&A, 494 527

Jarosik, N., Bennett, C. L., Dunkley, J., et al. 2011, ApJS, 192, 14

Leahy, J. P., Black, A. R. S., Dennett-Thorpe, J., et al. 1997, MNRAS, 291, 20

Massardi, M., Bonaldi, A., Bonavera, L., et al. 2011, MNRAS, 415, 1579

Mennella, A., Butler, R. C., Curto, A., et al. 2011, A\&A, 536, A3

Murphy, T., Sadler, E. M., Ekers, R. D., et al. 2010, MNRAS, 402, 2403

Perley, R. A., Chandler, C. J., Butler, B. J., \& Wrobel, J. M. 2011, ApJ, 739, L1

Planck Collaboration 2011a, A\&A, 536, A1

Planck Collaboration 2011b, A\&A, 536, A7

Planck Collaboration 2011c, A\&A, 536, A13

Planck Collaboration 2011d, A\&A, 536, A14

Planck Collaboration 2011e, A\&A, 536, A15

Procopio, P., Massardi, M., Righini, S., et al. 2011, MNRAS, 417, 1123

Sadler, E. M., Ricci, R., Ekers, R. D., et al. 2006, MNRAS, 371, 898

Sajina, A., Partridge, B., Evans, T., et al. 2011, ApJ, 732, 45 
N. Kurinsky et al.: VLA/JVLA monitoring of bright northern radio sources

Table 3. VLA/JVLA source coordinates and flux densities.

\begin{tabular}{|c|c|c|c|c|c|c|c|}
\hline Source & $\begin{array}{c}\text { RA } \\
\text { J2000 }\end{array}$ & $\begin{array}{c}\text { Dec } \\
\text { J2000 }\end{array}$ & Dateobs & $\begin{array}{c}S_{C} \\
{[\mathrm{mJy}]}\end{array}$ & $\underset{[\mathrm{mJy}]}{S_{X}}$ & $\begin{array}{c}S_{K a} \\
{[\mathrm{mJy}]}\end{array}$ & $\underset{[\mathrm{mJy}]}{S_{Q}}$ \\
\hline J0006-0623 & 1.5578871 & -6.3931487 & 20100702 & $2415.0 \pm 9.3$ & $2301.6 \pm 14.9$ & $1653.4 \pm 8.2$ & $1369.1 \pm 10.0$ \\
\hline J0019+7327 & 4.9407767 & 73.4583381 & 20090827 & $1191.9 \pm 0.7$ & $1224.7 \pm 1.4$ & $* 1051.2 \pm 4.0$ & $901.9 \pm 6.2$ \\
\hline J0050-0929 & 12.6721558 & -9.4847806 & 20100723 & $254.1 \pm 1.0$ & $209.9 \pm 1.4$ & $123.7 \pm 0.6$ & $115.2 \pm 0.9$ \\
\hline J0108+0135 & 17.1615462 & 1.5834214 & 20100723 & $3558.8 \pm 13.6$ & $3909.3 \pm 25.4$ & $2736.1 \pm 13.6$ & $2295.3 \pm 16.8$ \\
\hline J0125-0005 & 21.3701825 & -0.0988699 & 20100803 & $1263.6 \pm 4.8$ & - & $787.1 \pm 3.9$ & $649.7 \pm 4.8$ \\
\hline J0137+3309 & 24.4208333 & 33.1597222 & 20090827 & $5537.1 \pm 0.1$ & $3267.0 \pm 0.1$ & $* 1234.9 \pm 0.1$ & $635.4 \pm 0.1$ \\
\hline J0137+3309 & 24.4220808 & 33.1597592 & 20091115 & $5543.7 \pm 0.4$ & $3280.7 \pm 0.3$ & $* 1266.6 \pm 5.7$ & $667.1 \pm 0.5$ \\
\hline J0137+3309 & 24.4220808 & 33.1597592 & 20100702 & $5440.2 \pm 20.8$ & $3278.5 \pm 21.3$ & $858.1 \pm 4.3$ & $670.7 \pm 4.9$ \\
\hline J0137+3309 & 24.4220808 & 33.1597592 & 20100723 & $5438.7 \pm 20.8$ & - & $858.9 \pm 4.3$ & $669.5 \pm 4.9$ \\
\hline J0137+3309 & 24.4220808 & 33.1597592 & 20100803 & $5434.5 \pm 20.8$ & - & $858.1 \pm 4.3$ & $669.8 \pm 4.9$ \\
\hline J0137+3309 & 24.4220808 & 33.1597592 & 20100907 & $5428.6 \pm 20.8$ & $3279.9 \pm 21.3$ & $858.4 \pm 4.3$ & $670.6 \pm 4.9$ \\
\hline J0137+3309 & 24.4220808 & 33.1597592 & 20100920 & $5440.3 \pm 21.0$ & - & $858.8 \pm 4.3$ & $666.3 \pm 4.9$ \\
\hline J0137+3309 & 24.4220808 & 33.1597592 & 20100924 & $5431.0 \pm 21.2$ & - & $858.5 \pm 4.3$ & $684.7 \pm 5.0$ \\
\hline J0137+3309 & 24.4220808 & 33.1597592 & 20101020 & $5434.2 \pm 21.2$ & - & $860.7 \pm 4.3$ & $672.6 \pm 5.0$ \\
\hline J0137+3309 & 24.4220808 & 33.1597592 & 20101109 & $5392.7 \pm 24.5$ & - & $860.5 \pm 4.5$ & $672.7 \pm 5.0$ \\
\hline J0137+3309 & 24.4220808 & 33.1597592 & 20101120 & $5403.8 \pm 21.9$ & - & $858.9 \pm 4.3$ & $674.7 \pm 5.0$ \\
\hline J0137+3309 & 24.4220808 & 33.1597592 & 20101127 & $5385.6 \pm 23.5$ & $3316.1 \pm 23.0$ & $861.8 \pm 4.4$ & $674.0 \pm 5.0$ \\
\hline J0217+7349 & 34.3783892 & 73.8257283 & 20090827 & $4253.2 \pm 1.0$ & $4255.6 \pm 4.2$ & $* 2974.5 \pm 14.2$ & $2169.1 \pm 17.8$ \\
\hline $\mathrm{J} 0217+7349$ & 34.3783892 & 73.8257283 & 20100920 & $3874.4 \pm 14.9$ & - & $2244.3 \pm 11.1$ & $1922.5 \pm 14.1$ \\
\hline J0217+7349 & 34.3783892 & 73.8257283 & 20100924 & - & - & $2284.8 \pm 11.3$ & $2284.6 \pm 16.7$ \\
\hline $\mathrm{J} 0228+6721$ & 37.2085479 & 67.3508415 & 20100920 & $1108.8 \pm 4.3$ & - & $903.6 \pm 4.5$ & $861.8 \pm 6.3$ \\
\hline J0319+4130 & 49.9506671 & 41.5116953 & 20100907 & $14555.1 \pm 56.1$ & $21103.0 \pm 137.0$ & $18627.6 \pm 92.4$ & $16539.0 \pm 121.0$ \\
\hline $\mathrm{J} 0336+3218$ & 54.1254483 & 32.3081507 & 20100907 & - & $2095.6 \pm 13.6$ & $2843.4 \pm 14.1$ & $2571.6 \pm 18.8$ \\
\hline $\mathrm{J} 0359+5057$ & 59.8739471 & 50.9639337 & 20100920 & $8834.3 \pm 33.8$ & - & $8246.9 \pm 40.9$ & $7162.9 \pm 52.4$ \\
\hline J0418+3801 & 64.5886542 & 38.0266111 & 20100920 & $2827.4 \pm 19.6$ & - & $2233.3 \pm 11.1$ & $2077.3 \pm 15.2$ \\
\hline J0423-0120 & 65.8125000 & -1.3425000 & 20091103 & - & - & $* 8505.0 \pm 0.1$ & $9245.7 \pm 0.1$ \\
\hline J0423-0120 & 65.8157917 & -1.3426111 & 20091103 & $3966.9 \pm 0.5$ & $4398.9 \pm 0.5$ & $* 8585.7 \pm 0.6$ & $9246.5 \pm 0.6$ \\
\hline J0423-0120 & 65.8158363 & -1.3425181 & 20100907 & - & $6200.0 \pm 40.2$ & $5651.5 \pm 28.0$ & $5183.3 \pm 37.9$ \\
\hline $\mathrm{J} 0433+0521$ & 68.2962312 & 5.3543387 & 20100907 & $3225.1 \pm 12.4$ & $2765.8 \pm 18.0$ & $1539.1 \pm 7.7$ & $1333.7 \pm 9.8$ \\
\hline J0449+1121 & 72.2819629 & 11.3579435 & 20100907 & $1175.2 \pm 4.5$ & $1323.4 \pm 8.6$ & $1254.8 \pm 6.2$ & $1126.1 \pm 8.2$ \\
\hline $\mathrm{J} 0449+1121$ & 72.2819629 & 11.3579435 & 20100920 & $1161.5 \pm 4.5$ & - & $1248.4 \pm 6.2$ & $1173.3 \pm 8.6$ \\
\hline J0501-0159 & 75.3375000 & -1.9872934 & 20100907 & $1037.5 \pm 4.0$ & $1158.7 \pm 7.5$ & $1497.5 \pm 7.4$ & $1382.2 \pm 10.1$ \\
\hline $\mathrm{J} 0510+1800$ & 77.5098713 & 18.0115505 & 2010920 & - & - & $565.3 \pm 2.8$ & $584.6 \pm 4.3$ \\
\hline $\mathrm{J} 0530+1331$ & 82.7350696 & 13.5319860 & 20100920 & $2191.4 \pm 8.4$ & - & $1119.8 \pm 5.6$ & $1098.3 \pm 8.0$ \\
\hline $\mathrm{J} 0530+1331$ & 82.7350696 & 13.5319860 & 20100924 & $2247.6 \pm 8.6$ & - & $1140.4 \pm 5.7$ & $1239.2 \pm 9.1$ \\
\hline J0541-0541 & 85.4086808 & -5.6970634 & 20101130 & $2035.6 \pm 1.0$ & $1046.7 \pm 1.0$ & $582.0 \pm 0.2$ & - \\
\hline J0555+3948 & 88.8783567 & 39.8136569 & 20100301 & 5338.6 & 4835.9 & 2681.1 & 2639.4 \\
\hline J0555+3948 & 88.8783567 & 39.8136569 & 20100924 & $5199.3 \pm 19.9$ & - & $2453.9 \pm 12.2$ & $2461.5 \pm 18.0$ \\
\hline J0555+3948 & 88.8783567 & 39.8136569 & 20101018 & $5337.5 \pm 0.3$ & - & $2682.4 \pm 0.7$ & $2622.3 \pm 1.7$ \\
\hline J0555+3948 & 88.8783567 & 39.8136569 & 20101130 & $5069.1 \pm 8.8$ & $4738.7 \pm 4.6$ & $2681.1 \pm 0.4$ & $2635.9 \pm 0.6$ \\
\hline J0607-0834 & 91.9987467 & -8.5805495 & 20100924 & - & - & $2237.8 \pm 11.1$ & $2235.1 \pm 16.4$ \\
\hline $\mathrm{J} 0646+4451$ & 101.6334417 & 44.8546084 & 20100924 & - & - & $2532.5 \pm 12.6$ & $2537.1 \pm 18.6$ \\
\hline $\mathrm{J} 0721+7120$ & 110.4727021 & 71.3434343 & 20101018 & $1738.2 \pm 2.6$ & - & $4024.2 \pm 1.3$ & $4251.5 \pm 2.2$ \\
\hline J0725-0054 & 111.4610000 & -0.9156944 & 20091103 & - & - & $* 2571.0 \pm 0.1$ & $2365.0 \pm 0.1$ \\
\hline J0725-0054 & 111.4610000 & -0.9157068 & 20101018 & $3451.3 \pm 1.7$ & $4073.0 \pm 0.5$ & $4045.8 \pm 1.2$ & $4093.5 \pm 0.7$ \\
\hline $\mathrm{J} 0738+1742$ & 114.5308071 & 17.7052773 & 20101018 & $797.5 \pm 1.8$ & $784.1 \pm 0.7$ & $580.8 \pm 0.3$ & $591.2 \pm 0.8$ \\
\hline $\mathrm{J} 0739+0137$ & 114.8251408 & 1.6179494 & 20091022 & $1143.0 \pm 10.0$ & $1260.0 \pm 1.3$ & $* 1546.7 \pm 4.1$ & $1652.8 \pm 3.2$ \\
\hline $\mathrm{J} 0739+0137$ & 114.8251412 & 1.6179494 & 20101018 & $833.7 \pm 1.1$ & $866.2 \pm 0.4$ & $993.6 \pm 0.5$ & $1042.9 \pm 0.7$ \\
\hline J0745-0044 & 116.4750000 & -0.7380556 & 20091103 & $1970.0 \pm 0.1$ & $1964.0 \pm 0.1$ & $* 960.0 \pm 0.1$ & $605.0 \pm 0.1$ \\
\hline $\mathrm{J} 0750+1231$ & 117.7166667 & 12.5177778 & 20091103 & $3685.0 \pm 0.1$ & $4486.0 \pm 0.1$ & $* 4400.0 \pm 0.1$ & $3940.0 \pm 0.1$ \\
\hline $\mathrm{J} 0750+1231$ & 117.7168571 & 12.5180078 & 20101018 & $3750.8 \pm 0.5$ & - & $3079.0 \pm 0.9$ & $3002.3 \pm 1.4$ \\
\hline J0757+0956 & 119.2776667 & 9.9430278 & 20091022 & $1143.0 \pm 6.0$ & $1530.0 \pm 1.2$ & $* 1903.2 \pm 4.1$ & $1964.7 \pm 5.2$ \\
\hline $\mathrm{J} 0757+0956$ & 119.2776787 & 9.9430145 & 20101018 & $1026.5 \pm 0.3$ & $1185.0 \pm 1.2$ & $1250.4 \pm 0.4$ & $1276.4 \pm 1.3$ \\
\hline $\mathrm{J} 0808+4950$ & 122.1652758 & 49.8434806 & 20091022 & $464.6 \pm 0.9$ & $454.0 \pm 0.4$ & $* 488.0 \pm 1.3$ & $4220.6 \pm 2.1$ \\
\hline J0825+0309 & 126.4595833 & 3.1566667 & 20091022 & $776.9 \pm 0.7$ & $737.4 \pm 0.6$ & $* 682.1 \pm 3.5$ & $689.7 \pm 2.3$ \\
\hline J0825+0309 & 126.4597433 & 3.1568111 & 20101108 & $559.6 \pm 5.4$ & - & $925.8 \pm 2.4$ & $1005.9 \pm 1.1$ \\
\hline $\mathrm{J} 0830+2410$ & 127.7170000 & 24.1833333 & 20091022 & $1269.0 \pm 7.0$ & $1246.0 \pm 1.0$ & $* 1072.7 \pm 5.1$ & $877.4 \pm 4.2$ \\
\hline $\mathrm{J} 0841+7053$ & 130.3515417 & 70.8950556 & 20091022 & $1762.0 \pm 9.0$ & $1484.5 \pm 0.8$ & $* 2267.0 \pm 4.7$ & $2900.0 \pm 9.4$ \\
\hline $\mathrm{J} 0841+7053$ & 130.3515221 & 70.8950481 & 20101018 & $1872.7 \pm 0.6$ & - & $2905.8 \pm 0.9$ & $2559.4 \pm 1.5$ \\
\hline J0854+2006 & 133.7036454 & 20.1085114 & 20101108 & $3531.4 \pm 33.9$ & - & $5997.9 \pm 15.4$ & $6803.7 \pm 7.1$ \\
\hline $\mathrm{J} 0909+4253$ & 137.3895833 & 42.8961389 & 20091118 & $1772.6 \pm 0.6$ & $1371.1 \pm 1.1$ & $* 973.5 \pm 1.6$ & $817.1 \pm 2.2$ \\
\hline J0920+4441 & 140.2435771 & 44.6983292 & 20091118 & $1124.5 \pm 0.4$ & $1518.3 \pm 1.0$ & $* 2475.3 \pm 0.6$ & $2393.0 \pm 1.0$ \\
\hline $\mathrm{J} 0920+4441$ & 140.2435771 & 44.6983292 & 20091118 & $1130.2 \pm 0.5$ & $1530.5 \pm 0.6$ & $* 2478.5 \pm 1.7$ & $2495.2 \pm 5.5$ \\
\hline $\mathrm{J} 0920+4441$ & 140.2435771 & 44.6983292 & 20101108 & $1138.9 \pm 10.9$ & - & $2758.0 \pm 7.1$ & $3251.8 \pm 3.5$ \\
\hline J0927+3902 & 141.7625579 & 39.0391255 & 20091118 & $11092.0 \pm 3.5$ & $11846.0 \pm 6.7$ & $* 9388.5 \pm 40.9$ & $7460.0 \pm 31.9$ \\
\hline
\end{tabular}


Table 3. continued.

\begin{tabular}{|c|c|c|c|c|c|c|c|}
\hline Source & $\begin{array}{c}\text { RA } \\
\text { J2000 }\end{array}$ & $\begin{array}{c}\text { Dec } \\
\text { J2000 }\end{array}$ & Dateobs & $\begin{array}{c}S_{C} \\
{[\mathrm{mJy}]}\end{array}$ & $\begin{array}{c}S_{X} \\
{[\mathrm{mJy}]}\end{array}$ & $\begin{array}{c}S_{K a} \\
{[\mathrm{mJy}]}\end{array}$ & $\begin{array}{c}S_{Q} \\
{[\mathrm{mJy}]}\end{array}$ \\
\hline J0927+3902 & 141.7625579 & 39.0391255 & 20101108 & $10441.0 \pm 100.8$ & - & $9309.7 \pm 23.9$ & $9996.1 \pm 10.5$ \\
\hline J0948+4039 & 147.2305754 & 40.6623853 & 20091118 & $1656.3 \pm 0.8$ & $1563.0 \pm 1.3$ & $* 1430.0 \pm 20.0$ & $1130.0 \pm 10.0$ \\
\hline J0948+4039 & 147.2305754 & 40.6623853 & 20101108 & $1772.0 \pm 19.5$ & - & $1151.7 \pm 3.0$ & $1293.9 \pm 1.5$ \\
\hline $\mathrm{J} 0956+2515$ & 149.2078142 & 25.2544583 & 20101108 & $667.0 \pm 6.3$ & - & $1230.9 \pm 3.2$ & $1316.2 \pm 1.5$ \\
\hline J0956+2515 & 149.2078142 & 25.2544583 & 20101130 & $642.6 \pm 1.2$ & $699.9 \pm 0.8$ & $1468.7 \pm 0.2$ & $1757.4 \pm 0.6$ \\
\hline $\mathrm{J} 1038+0512$ & 159.6949167 & 5.2080806 & 20100103 & $1233.0 \pm 29.0$ & $1920.0 \pm 1.3$ & $* 1513.0 \pm 3.5$ & $1164.2 \pm 3.6$ \\
\hline $\mathrm{J} 1043+2408$ & 160.7876492 & 24.1431693 & 20101130 & $644.5 \pm 0.2$ & $742.9 \pm 1.0$ & $1149.2 \pm 0.2$ & $1299.0 \pm 0.5$ \\
\hline $\mathrm{J} 1058+0234$ & 164.6208333 & 2.5830556 & 20091103 & - & - & $* 5985.0 \pm 0.1$ & $6344.0 \pm 0.1$ \\
\hline $\mathrm{J} 1058+0133$ & 164.6233542 & 1.5663333 & 20100103 & $3193.0 \pm 7.0$ & $4186.0 \pm 3.0$ & $* 5431.9 \pm 13.3$ & $5404.0 \pm 24.5$ \\
\hline $\mathrm{J} 1130+3815$ & 172.7220000 & 38.2551389 & 20100103 & $1334.0 \pm 2.0$ & $1356.0 \pm 1.0$ & $* 1041.0 \pm 1.5$ & $723.7 \pm 3.6$ \\
\hline $\mathrm{J} 1130+3815$ & 172.7220108 & 38.2551520 & 20101130 & $1370.6 \pm 2.7$ & $1538.7 \pm 1.7$ & $1327.8 \pm 0.4$ & $1319.5 \pm 0.5$ \\
\hline $\mathrm{J} 1153+8043$ & 178.3500000 & 80.7311111 & 20091103 & - & - & $* 803.0 \pm 0.1$ & $642.0 \pm 0.1$ \\
\hline $\mathrm{J} 1153+4931$ & 178.3519583 & 49.5191194 & 20100103 & $1167.0 \pm 1.2$ & $949.0 \pm 0.9$ & $* 904.1 \pm 0.8$ & $849.2 \pm 2.4$ \\
\hline $\mathrm{J} 1159+2914$ & 179.8791667 & 29.2452778 & 20091103 & $1609.0 \pm 0.1$ & $1530.0 \pm 0.1$ & $* 1416.0 \pm 0.1$ & $1140.0 \pm 0.1$ \\
\hline $\mathrm{J} 1159+2914$ & 179.8826250 & 29.2455556 & 20100103 & $1609.0 \pm 1.3$ & $1530.0 \pm 3.0$ & $* 1446.6 \pm 4.1$ & $1214.3 \pm 9.6$ \\
\hline $\mathrm{J} 1221+2813$ & 185.3820438 & 28.2329168 & 201074 & $446.6 \pm 4.2$ & $419.3 \pm 2.2$ & $326.7 \pm 0.8$ & $297.5 \pm 0.4$ \\
\hline $\mathrm{J} 1222+0413$ & 185.5939567 & 4.2210490 & 20100704 & $783.7 \pm 7.4$ & $1076.3 \pm 5.7$ & $1000.8 \pm 2.6$ & $903.6 \pm 1.1$ \\
\hline $\mathrm{J} 1222+0413$ & 185.5939567 & 4.2210490 & 20100709 & $782.8 \pm 7.4$ & $1089.7 \pm 5.7$ & $1063.5 \pm 2.7$ & $1021.7 \pm 1.1$ \\
\hline $\mathrm{J} 1224+2122$ & 186.2271250 & 21.3797222 & 20100103 & $1233.0 \pm 2.0$ & $1267.0 \pm 1.4$ & $* 1442.5 \pm 4.1$ & $1260.0 \pm 8.5$ \\
\hline $\mathrm{J} 1229+0203$ & 187.2779154 & 2.0523884 & 20100704 & $37172.1 \pm 360.1$ & $31724.1 \pm 187.0$ & $22899.1 \pm 59.9$ & $19226.6 \pm 24.5$ \\
\hline $\mathrm{J} 1229+0203$ & 187.2779154 & 2.0523884 & 20100709 & $37738.1 \pm 357.1$ & $32793.1 \pm 173.6$ & $24318.3 \pm 62.4$ & $19812.5 \pm 20.3$ \\
\hline $\mathrm{J} 1230+1223$ & 187.7059308 & 12.3911233 & 20100704 & $53260.1 \pm 548.8$ & $24892.1 \pm 163.9$ & $1669.4 \pm 14.2$ & $1807.8 \pm 7.7$ \\
\hline $\mathrm{J} 1230+1223$ & 187.7059308 & 12.3911233 & 20100709 & $59530.1 \pm 719.9$ & $26430.1 \pm 177.1$ & $1913.7 \pm 13.5$ & $1884.1 \pm 5.0$ \\
\hline $\mathrm{J} 1310+3220$ & 197.6194167 & 32.3455000 & 20100103 & $1427.0 \pm 1.7$ & $2133.0 \pm 1.4$ & $* 2917.7 \pm 5.1$ & $2125.2 \pm 16.0$ \\
\hline $\mathrm{J} 1310+3220$ & 197.6194325 & 32.3454953 & 20100709 & $1963.4 \pm 18.5$ & $2718.1 \pm 14.3$ & $2548.0 \pm 6.5$ & $2312.3 \pm 2.4$ \\
\hline $\mathrm{J} 1327+2210$ & 201.7535879 & 22.1806010 & 20100709 & $1856.9 \pm 17.5$ & $1746.1 \pm 9.2$ & $1232.9 \pm 3.2$ & $1077.2 \pm 1.1$ \\
\hline $\mathrm{J} 1331+3030$ & 202.7845333 & 30.5091553 & 20091118 & $7481.4 \pm 0.6$ & $5202.6 \pm 0.5$ & $* 2574.5 \pm 1.6$ & $1557.1 \pm 0.5$ \\
\hline $\mathrm{J} 1331+3030$ & 202.7845333 & 30.5091553 & 20100103 & $7485.0 \pm 0.5$ & $5202.3 \pm 0.4$ & $* 2570.4 \pm 0.3$ & $1532.5 \pm 0.3$ \\
\hline $\mathrm{J} 1331+3030$ & 202.7845333 & 30.5091553 & 20100704 & $7398.4 \pm 69.8$ & $5200.4 \pm 27.3$ & $1890.9 \pm 4.9$ & $1530.3 \pm 1.7$ \\
\hline $\mathrm{J} 1331+3030$ & 202.7845333 & 30.5091553 & 20100709 & $7387.3 \pm 69.7$ & $5243.5 \pm 27.5$ & $1891.3 \pm 4.9$ & $1531.1 \pm 1.6$ \\
\hline $\mathrm{J} 1331+3030$ & 202.7845333 & 30.5091553 & 20100915 & $7382.4 \pm 69.7$ & $5251.5 \pm 27.6$ & $1898.6 \pm 4.9$ & $1533.8 \pm 1.7$ \\
\hline $\mathrm{J} 1331+3030$ & 202.7845333 & 30.5091553 & 20101108 & $7251.2 \pm 68.7$ & - & $1892.2 \pm 4.9$ & $1532.6 \pm 1.6$ \\
\hline $\mathrm{J} 1419+5423$ & 214.9441667 & 54.3872222 & 20100103 & $1178.0 \pm 1.6$ & $1249.0 \pm 7.0$ & $* 1257.6 \pm 1.6$ & $1251.5 \pm 4.3$ \\
\hline $\mathrm{J} 1419+5423$ & 214.9441558 & 54.3874409 & 20100704 & $1097.6 \pm 10.4$ & $1096.9 \pm 5.8$ & $963.6 \pm 2.5$ & $949.8 \pm 1.1$ \\
\hline $\mathrm{J} 1419+5423$ & 214.9441558 & 54.3874409 & 20100709 & $1063.2 \pm 10.1$ & $1081.2 \pm 5.7$ & $909.7 \pm 2.3$ & $893.3 \pm 0.9$ \\
\hline $\mathrm{J} 1642+6856$ & 250.5327021 & 68.9443768 & 20100704 & $2520.8 \pm 23.8$ & $2617.4 \pm 13.7$ & $1696.2 \pm 4.9$ & $1524.8 \pm 2.1$ \\
\hline $\mathrm{J} 1642+6856$ & 250.5327021 & 68.9443768 & 20100709 & $2482.9 \pm 23.4$ & - & $1735.3 \pm 4.5$ & $1567.7 \pm 1.6$ \\
\hline $\mathrm{J} 1728+1215$ & 262.0293800 & 12.2609683 & 2010915 & - & $464.8 \pm 2.4$ & $451.8 \pm 1.2$ & $425.5 \pm 0.5$ \\
\hline J1743-0350 & 265.9952337 & -3.8346158 & 20100915 & $3316.3 \pm 31.3$ & $3469.5 \pm 18.2$ & $3616.1 \pm 9.3$ & $3298.0 \pm 3.5$ \\
\hline $\mathrm{J} 1751+0939$ & 267.8867442 & 9.6502024 & 20100915 & $1619.8 \pm 15.3$ & $1905.7 \pm 10.0$ & $2111.2 \pm 5.5$ & $2085.1 \pm 2.2$ \\
\hline $\mathrm{J} 1800+3848$ & 270.1031892 & 38.8085271 & 20100915 & - & - & $551.6 \pm 1.4$ & $504.8 \pm 0.6$ \\
\hline $\mathrm{J} 1800+7828$ & 270.1903496 & 78.4677829 & 20101020 & $2497.1 \pm 9.6$ & - & $2522.4 \pm 12.5$ & $2515.8 \pm 18.4$ \\
\hline J1806+6949 & 271.7111692 & 69.8244746 & 20100704 & $1537.1 \pm 14.7$ & $1478.1 \pm 7.8$ & $1202.0 \pm 3.5$ & $1185.5 \pm 1.9$ \\
\hline J1806+6949 & 271.7111692 & 69.8244746 & 20100709 & $1524.5 \pm 14.6$ & $1481.1 \pm 7.8$ & $1235.2 \pm 3.4$ & $1192.5 \pm 1.8$ \\
\hline $\mathrm{J} 1806+6949$ & 271.7111692 & 69.8244746 & 20101109 & $1383.4 \pm 6.4$ & - & $1241.0 \pm 6.3$ & $1296.0 \pm 9.5$ \\
\hline $\mathrm{J} 1806+6949$ & 271.7111692 & 69.8244746 & 20101127 & $1440.8 \pm 6.3$ & $1409.2 \pm 10.3$ & $1058.7 \pm 5.3$ & $1231.3 \pm 9.1$ \\
\hline $\mathrm{J} 1824+5651$ & 276.0294517 & 56.8504141 & 20100915 & - & $1362.2 \pm 7.2$ & $1137.2 \pm 3.1$ & $1096.9 \pm 1.4$ \\
\hline $\mathrm{J} 1829+4844$ & 277.3824300 & 48.7461558 & 20100915 & $5042.5 \pm 47.6$ & $3716.4 \pm 19.5$ & $2498.6 \pm 6.8$ & $2405.8 \pm 4.4$ \\
\hline $\mathrm{J} 1849+6705$ & 282.3169679 & 67.0949111 & 20100723 & $1434.7 \pm 5.5$ & $2152.3 \pm 14.0$ & $2720.4 \pm 13.5$ & $2473.9 \pm 18.1$ \\
\hline $\mathrm{J} 1849+6705$ & 282.3169679 & 67.0949111 & 20100803 & $1452.0 \pm 5.7$ & - & $2979.9 \pm 14.8$ & $2861.2 \pm 20.9$ \\
\hline $\mathrm{J} 1849+6705$ & 282.3169679 & 67.0949111 & 20100915 & $1395.9 \pm 13.2$ & $2050.6 \pm 10.8$ & $2463.8 \pm 6.3$ & $2212.1 \pm 2.3$ \\
\hline $\mathrm{J} 1849+6705$ & 282.3169679 & 67.0949111 & 20101020 & - & - & $2252.6 \pm 11.2$ & $2349.2 \pm 17.2$ \\
\hline $\mathrm{J} 1849+6705$ & 282.3169679 & 67.0949111 & 20101109 & $1259.9 \pm 5.0$ & - & $2247.8 \pm 11.2$ & $2295.2 \pm 16.8$ \\
\hline $\mathrm{J} 1927+7358$ & 291.9520633 & 73.9671028 & 20101020 & - & - & $4446.9 \pm 22.2$ & $4851.7 \pm 35.5$ \\
\hline $\mathrm{J} 1955+5131$ & 298.9280763 & 51.5301517 & 20101020 & - & - & $1333.4 \pm 6.6$ & $1589.4 \pm 11.6$ \\
\hline $\mathrm{J} 1955+5131$ & 298.9280763 & 51.5301517 & 20101120 & $1296.5 \pm 5.7$ & - & $1267.8 \pm 6.3$ & $1127.7 \pm 8.3$ \\
\hline $\mathrm{J} 1955+5131$ & 298.9280763 & 51.5301517 & 20101127 & $1326.8 \pm 5.9$ & $1387.5 \pm 9.2$ & $1141.8 \pm 5.7$ & $1015.4 \pm 7.4$ \\
\hline $\mathrm{J} 2005+7752$ & 301.3791604 & 77.8786799 & 20101020 & $924.7 \pm 3.6$ & - & $689.6 \pm 3.4$ & $701.0 \pm 5.2$ \\
\hline J2007+4029 & 301.9372704 & 40.4968345 & 20091115 & $2956.5 \pm 3.1$ & $3810.3 \pm 1.9$ & $* 3141.2 \pm 14.7$ & $2465.4 \pm 20.3$ \\
\hline $\mathrm{J} 2007+4029$ & 301.9372704 & 40.4968345 & 20101020 & $3534.2 \pm 13.6$ & - & $3506.0 \pm 17.4$ & $3949.9 \pm 29.0$ \\
\hline $\mathrm{J} 2022+6136$ & 305.5278404 & 61.6163346 & 20100702 & $3161.5 \pm 12.1$ & $3058.8 \pm 19.9$ & $1136.0 \pm 5.6$ & $839.6 \pm 6.1$ \\
\hline $\mathrm{J} 2022+6136$ & 305.5278404 & 61.6163346 & 20100723 & $3227.4 \pm 12.4$ & $3089.9 \pm 20.1$ & $1134.5 \pm 5.6$ & $819.8 \pm 6.0$ \\
\hline $\mathrm{J} 2022+6136$ & 305.5278404 & 61.6163346 & 20100803 & $3242.4 \pm 12.4$ & - & $1213.7 \pm 6.0$ & $1027.5 \pm 7.5$ \\
\hline $\mathrm{J} 2053+5427$ & 313.4791667 & 54.4597222 & 20091103 & $2850.0 \pm 0.1$ & $3311.0 \pm 0.1$ & $* 2460.0 \pm 0.1$ & $1360.0 \pm 0.1$ \\
\hline $\mathrm{J} 2123+0535$ & 320.9354892 & 5.5894703 & 20091115 & $2168.4 \pm 4.0$ & $1927.0 \pm 2.0$ & $* 1144.8 \pm 6.5$ & $847.5 \pm 9.3$ \\
\hline $\mathrm{J} 2123+0535$ & 320.9354892 & 5.5894703 & 20101109 & $2564.6 \pm 10.9$ & - & $1421.1 \pm 7.1$ & $1550.6 \pm 11.3$ \\
\hline
\end{tabular}


Table 3. continued.

\begin{tabular}{|c|c|c|c|c|c|c|c|}
\hline Source & $\begin{array}{c}\text { RA } \\
\text { J2000 }\end{array}$ & $\begin{array}{c}\text { Dec } \\
\text { J2000 }\end{array}$ & Dateobs & $\begin{array}{c}S_{C} \\
{[\mathrm{mJy}]}\end{array}$ & $\begin{array}{c}S_{X} \\
{[\mathrm{mJy}]}\end{array}$ & $\begin{array}{c}S_{K a} \\
{[\mathrm{mJy}]}\end{array}$ & $\begin{array}{c}S_{Q} \\
{[\mathrm{mJy}]}\end{array}$ \\
\hline $\mathrm{J} 2133+1443$ & 323.4057888 & 14.7295753 & 20101109 & $164.5 \pm 0.7$ & - & $133.7 \pm 0.7$ & $118.6 \pm 0.9$ \\
\hline J2134-0153 & 323.5429583 & -1.8881111 & 20091103 & - & - & $* 1925.5 \pm 0.1$ & $1545.0 \pm 0.1$ \\
\hline J2134-0153 & 323.5429583 & -1.8881111 & 20091115 & $2450.2 \pm 5.4$ & $2492.0 \pm 2.7$ & $* 2089.5 \pm 10.1$ & $1717.1 \pm 14.6$ \\
\hline $\mathrm{J} 2136+0041$ & 324.1607762 & 0.6983926 & 20091115 & - & $8334.4 \pm 35.4$ & $* 4367.0 \pm 27.2$ & $2536.1 \pm 26.3$ \\
\hline $\mathrm{J} 2136+0041$ & 324.1607762 & 0.6983926 & 20101109 & $9412.6 \pm 36.8$ & - & $4968.0 \pm 24.7$ & $5028.3 \pm 36.8$ \\
\hline $\mathrm{J} 2139+1423$ & 324.7554554 & 14.3933311 & 20091115 & $2986.9 \pm 5.0$ & $3202.7 \pm 2.1$ & $* 2080.3 \pm 9.3$ & $1294.1 \pm 14.7$ \\
\hline $\mathrm{J} 2139+1423$ & 324.7554554 & 14.3933311 & 20101120 & $2930.4 \pm 11.9$ & - & $2015.1 \pm 10.0$ & $1595.0 \pm 11.7$ \\
\hline $\mathrm{J} 2139+1423$ & 324.7554554 & 14.3933311 & 20101127 & $2987.5 \pm 11.7$ & $3345.4 \pm 21.8$ & $1613.0 \pm 8.0$ & $1726.4 \pm 12.6$ \\
\hline $\mathrm{J} 2148+0657$ & 327.0227500 & 6.9607222 & 20091115 & $5356.3 \pm 15.3$ & $5559.4 \pm 7.6$ & $* 3852.2 \pm 27.2$ & $3172.3 \pm 33.0$ \\
\hline $\mathrm{J} 2148+6107$ & 327.0668558 & 61.1182883 & 20090827 & $1224.7 \pm 0.6$ & $996.1 \pm 1.3$ & $* 601.1 \pm 2.5$ & $330.0 \pm 4.2$ \\
\hline $\mathrm{J} 2202+4216$ & 330.6804167 & 42.2775000 & 20091103 & $3994.0 \pm 0.1$ & $3996.0 \pm 0.1$ & $* 3440.0 \pm 0.1$ & $3085.0 \pm 0.1$ \\
\hline $\mathrm{J} 2203+3145$ & 330.8124167 & 31.7606389 & 20091103 & $2165.0 \pm 0.1$ & $2495.0 \pm 0.1$ & $* 2576.0 \pm 0.1$ & $2841.0 \pm 0.1$ \\
\hline $\mathrm{J} 2203+3145$ & 330.8123992 & 31.7606305 & 20100702 & $1997.6 \pm 8.3$ & $2515.4 \pm 16.3$ & $2864.1 \pm 14.2$ & $2500.8 \pm 18.3$ \\
\hline $\mathrm{J} 2203+1725$ & 330.8620417 & 17.4300556 & 20091103 & $987.0 \pm 0.1$ & $1055.0 \pm 0.1$ & $* 1035.0 \pm 0.1$ & $1073.0 \pm 0.1$ \\
\hline $\mathrm{J} 2203+1725$ & 330.8620571 & 17.4300688 & 20101120 & $937.2 \pm 4.6$ & - & $1573.3 \pm 7.8$ & $1271.6 \pm 9.3$ \\
\hline $\mathrm{J} 2203+1725$ & 330.8620571 & 17.4300688 & 20101127 & $893.4 \pm 3.6$ & $1197.2 \pm 7.8$ & $1558.5 \pm 7.7$ & $1663.6 \pm 12.2$ \\
\hline J2218-0335 & 334.7168333 & -3.5935833 & 20091115 & $2254.1 \pm 3.9$ & $1875.0 \pm 1.6$ & $* 1214.3 \pm 4.8$ & $1021.5 \pm 8.2$ \\
\hline J2225-0457 & 336.4469167 & -4.9503889 & 20091115 & $7219.7 \pm 16.0$ & $7795.0 \pm 5.0$ & $* 5656.0 \pm 24.0$ & $3597.0 \pm 26.0$ \\
\hline J2229-0832 & 337.4170417 & -8.5484722 & 20091115 & $2085.4 \pm 4.5$ & $1767.6 \pm 1.2$ & $* 1234.3 \pm 5.4$ & $1239.3 \pm 9.3$ \\
\hline $\mathrm{J} 2230+6946$ & 337.6519571 & 69.7744658 & 20090827 & $743.1 \pm 0.4$ & $748.2 \pm 0.9$ & $* 572.7 \pm 3.3$ & $405.9 \pm 3.7$ \\
\hline $\mathrm{J} 2232+1143$ & 338.1517083 & 11.7308056 & 20091103 & $5594.0 \pm 0.1$ & $5827.0 \pm 0.1$ & $* 4407.0 \pm 0.1$ & $3606.0 \pm 0.1$ \\
\hline $\mathrm{J} 2236+2828$ & 339.0936250 & 28.4826111 & 20091103 & $1285.0 \pm 0.1$ & $1273.0 \pm 0.1$ & $* 1412.0 \pm 0.1$ & $1547.0 \pm 0.1$ \\
\hline $\mathrm{J} 2236+2828$ & 339.0936287 & 28.4826148 & 20100702 & $1595.7 \pm 6.1$ & $1434.7 \pm 9.3$ & $1595.7 \pm 7.9$ & $1541.4 \pm 11.3$ \\
\hline $\mathrm{J} 2253+1608$ & 343.4906250 & 16.1482222 & 20091103 & $9463.0 \pm 0.1$ & $8176.0 \pm 0.1$ & $* 11834.0 \pm 0.1$ & $21570.1 \pm 0.1$ \\
\hline $\mathrm{J} 2253+1608$ & 343.4906162 & 16.1482114 & 20100702 & $12572.6 \pm 48.2$ & - & $28126.0 \pm 139.5$ & $26563.1 \pm 194.2$ \\
\hline $\mathrm{J} 2316+1618$ & 349.1654112 & 16.3018731 & 201072 & $246.1 \pm 1.0$ & $198.1 \pm 1.3$ & $139.3 \pm 0.7$ & $129.6 \pm 1.0$ \\
\hline $\mathrm{J} 2327+0940$ & 351.8899192 & 9.6692952 & 20100702 & $1024.9 \pm 3.9$ & $1002.9 \pm 6.5$ & $990.4 \pm 4.9$ & $891.8 \pm 6.5$ \\
\hline $\mathrm{J} 2327+0940$ & 351.8899192 & 9.6692952 & 20100723 & $1010.1 \pm 3.9$ & $1012.2 \pm 6.6$ & $1123.2 \pm 5.6$ & $1040.7 \pm 7.6$ \\
\hline $\mathrm{J} 2327+0940$ & 351.8899192 & 9.6692952 & 20100803 & $998.8 \pm 3.8$ & - & $1317.3 \pm 6.5$ & $1300.0 \pm 9.5$ \\
\hline $\mathrm{J} 2354+4553$ & 358.5903346 & 45.8845101 & 20100723 & $1260.0 \pm 4.8$ & $1012.9 \pm 6.6$ & $492.5 \pm 2.4$ & $399.5 \pm 2.9$ \\
\hline $\mathrm{J} 2354+4553$ & 358.5903346 & 45.8845101 & 20100803 & $1280.6 \pm 4.9$ & - & $500.7 \pm 2.5$ & $409.8 \pm 3.0$ \\
\hline
\end{tabular}

Notes. The errors include statistical error as well as calibration uncertainty. ${ }^{*}$ ) Observations marked with * are VLA observations done at $K(22 \mathrm{GHz})$ instead of $\mathrm{Ka}(33 \mathrm{GHz})$. 
A\&A 549, A133 (2013)

Table 4. Matches of our VLA/JVLA sources with sources in the ERCSC catalog.

\begin{tabular}{|c|c|c|c|}
\hline J2000 name & Planck name & (J)VLA dateobs & Planck dateobs \\
\hline J0006-0623 & PLCKERC030 G093.49-66.62 & 20100702 & 20091221 \\
\hline J0108+0135 & PLCKERC030 G131.82-60.99 & 20100723 & 20100112 \\
\hline J0125-0005 & PLCKERC030 G141.26-61.77 & 20100803 & 20100115,20100116 \\
\hline J0137+3309 & PLCKERC030 G133.94-28.63 & $\begin{array}{l}20100702,20100723,20100803, \\
20100907,20100920,20100924, \\
20101020,20101109,20101127, \\
20101120,20091115,20090827\end{array}$ & 20100130, 20100131 \\
\hline J0217+7349 & PLCKERC030 G128.95+11.97 & $20100920,20100924,20090827$ & $\begin{array}{c}20090911,20090912,20100219, \\
20100220\end{array}$ \\
\hline J0228+6721 & PLCKERC030 G132.15+06.22 & 20100920 & 20090906, 20100217, 20100218 \\
\hline J0319+4130 & PLCKERC030 G150.59-13.25 & 20100907 & 20090829, 20100218, 20100219 \\
\hline J0336+3218 & PLCKERC030 G159.01-18.79 & 20100907 & 20090828, 20090829, 20100220 \\
\hline J0359+5057 & PLCKERC030 G150.36-01.65 & 20100920 & 20090907, 20090908, 20100225 \\
\hline J0418+3801 & PLCKERC030 G161.66-08.78 & 20100920 & $\begin{array}{c}20090906,20090907,20100227, \\
20100228\end{array}$ \\
\hline J0423-0120 & PLCKERC030 G195.26-33.13 & $20100907,20091103,20091103$ & $\begin{array}{c}20090828,20090829,20100226, \\
20100227\end{array}$ \\
\hline J0433+0521 & PLCKERC030 G190.40-27.42 & 20100907 & 20090901,20100301 \\
\hline $49+1121$ & LCKERC030 C & 20100907,20100920 & 20090906, \\
\hline J0501-0159 & PLCKERC030 G201.45-25.29 & 20100907 & 20090905,20090 \\
\hline $\mathrm{J} 0530+1331$ & PLCKERC030 G191.42-11.03 & 20100920, 20100924 & $\begin{array}{c}20090914,20090915,20100310, \\
20100311\end{array}$ \\
\hline J0555+3948 & PLCKERC030 G171.66+07.28 & $\begin{array}{c}20100301,20100924,20101018, \\
20101130\end{array}$ & 20090922, 20100313, 20100314 \\
\hline J0607-0834 & PLCKERC030 G215.75-13.51 & 20100924 & $\begin{array}{c}20090919,20090920,20100322, \\
20100323\end{array}$ \\
\hline J0646+4451 & PLCKERC030 G171.09+17.92 & 20100924 & 20090930, 20091001, 20100322 \\
\hline J0721+7120 & PLCKERC03 & 20101018 & 20091005, 20100317, 20100318 \\
\hline J0725-0054 & PLCKERC030 G217.69+07.21 & 20101018, 20091103 & 20091009, 20091010, 20100407 \\
\hline $\mathrm{J} 0738+1742$ & PLCKERC030 G201.84+18.14 & 20101018 & 20091010, 20091011, 20100406 \\
\hline J0739+0137 & PLCKERC030 G216.97+11.36 & 20101018, 20091022 & $\begin{array}{c}20091012,20091013,20100409, \\
20100410\end{array}$ \\
\hline J0745-0044 & PLCKERC030 G219.91+11.78 & 20091103 & $\begin{array}{c}20091014,20091015,20100411, \\
20100412\end{array}$ \\
\hline J0750+1231 & LCKERC030 G208.18+18.75 & & 20091014,20100410 \\
\hline & $11.33+19.05$ & 20101018, 2009 & 20091016, 20100411, 20100412 \\
\hline J0825+0309 & PLCKERC030 G221.26+22.36 & 20101108, 20091022 & $\begin{array}{c}20091024,20091025,20100419, \\
20100420\end{array}$ \\
\hline $\mathrm{J} 0830+2410$ & PLCKERC030 G200.06+31.89 & 20091022 & 20091021, 20100415, 20100416 \\
\hline $\mathrm{J} 0841+7053$ & PLCKERC030 G143.55+34.41 & 20101018, 20091022 & 20091012, 20100326, 20100327 \\
\hline J0854+2006 & PLCKERC030 G206.78+35.81 & 20101108 & $20091027,20100421,20100422$ \\
\hline J0909+4253 & PLCKERC030 G178.32+42.86 & 20091118 & 20091024,20100417 \\
\hline J0920+4441 & PLCKERC030 G175.71+44.81 & 20091118, 20091118, 20101108 & 20091025, 20100418, 20100419 \\
\hline J0927+3902 & PLCKERC030 G183.72+46.16 & 20091118,20101108 & 20091028, 20100422, 20100423 \\
\hline J0948+4039 & PLCKERC030 G181.02+50.31 & 20091118, 20101108 & 20091031, 20100426, 20100427 \\
\hline J0956+2515 & PLCKERC030 G & 2010110 & 20091108,20100505 \\
\hline $\mathrm{J} 1043+2408$ & 1.00 & & 20091119,2009 \\
\hline $\mathrm{J} 1058+0133$ & PLCKERC030 G251.59+52.70 & 20100103 & $\begin{array}{c}20091207,20091208,20100601, \\
20100602\end{array}$ \\
\hline $\mathrm{J} 1130+3815$ & PLCKERC030 G174.47+69.79 & 20101130, 20100103 & 20091121,20100527 \\
\hline $\mathrm{J} 1153+4931$ & PLCKERC030 G145.58+64.96 & 20100103 & $\begin{array}{c}20091116,20091117,20100525, \\
20100526\end{array}$ \\
\hline $\mathrm{J} 1153+8043$ & PLCKERC030 G125.75+35.85 & 20091103 & $\begin{array}{c}20091016,20091017,20100321, \\
20100322\end{array}$ \\
\hline J1159+2914 & PLCKERC030 G199.42+78.39 & & 20091204, 20091205 \\
\hline $\mathrm{J} 1222+0413$ & PLCKERC030 G284.63+66.05 & 20100704, 20100709 & 20091229, 20091230 \\
\hline $\mathrm{J} 1224+2122$ & PLCKERC030 G255.00+81.65 & 20100103 & 20091218, 20091219 \\
\hline $\mathrm{J} 1229+0203$ & PLCKERC030 G290.02+64.36 & 20100704, 20100709 & 20100102 \\
\hline $\mathrm{J} 1230+1223$ & PLCKERC030 G283.75+74.54 & 20100704, 20100709 & 20091226, 20091227 \\
\hline $\mathrm{J} 1310+3220$ & PLCKERC030 G085.86+83.31 & 20100709,20100103 & 20091221, 20091222 \\
\hline $\mathrm{J} 1327+2210$ & PLCKERC030 G003.62+80.50 & 20100709 & 20100105,20100106 \\
\hline $\mathrm{J} 1331+3030$ & PLCKERC030 G056.70+80.65 & $\begin{array}{l}20100704,20100709,20100915, \\
20101108,20091118,20100103\end{array}$ & 20091230, 20091231 \\
\hline $\mathrm{J} 1419+5423$ & PLCKERC030 G098.28+58.29 & $20100704,20100709,20100103$ & 20091206, 20091207 \\
\hline $\mathrm{J} 1642+6856$ & PLCKERC030 G100.69+36.62 & 20100704, 20100709 & $\begin{array}{l}20091106,20091107,20091108, \\
20100222,20100223,20100224,\end{array}$ \\
\hline
\end{tabular}


Table 4. continued.

\begin{tabular}{|c|c|c|c|}
\hline J2000 name & Planck name & (J)VLA dateobs & Planck dateobs \\
\hline & & & $\begin{array}{c}20100225,20100226,20100409, \\
20100410,20100411,20100412, \\
20100413,20100414\end{array}$ \\
\hline J1743-0350 & PLCKERC030 G021.60+13.15 & 20100915 & 20090914, 20100317 \\
\hline $\mathrm{J} 1751+0939$ & PLCKERC030 G034.92+17.63 & 20100915 & $20090913,20090914,20100321$ \\
\hline $\mathrm{J} 1800+3848$ & PLCKERC030 G065.15+26.02 & 20100915 & $20090907,20100329,20100330$ \\
\hline $\mathrm{J} 1800+7828$ & PLCKERC030 G110.05+29.07 & 20101020 & $\begin{array}{c}20091011,20091012,20100218, \\
20100219\end{array}$ \\
\hline J1806+6949 & PLCKERC030 G100.12+29.18 & $\begin{array}{c}20100704,20100709,20101109, \\
20101127\end{array}$ & $\begin{array}{l}\text { 20091022, 20091023, 20100129, } \\
\text { 20100130, 20100131, 20100511, } \\
20100512,20100513,20100514\end{array}$ \\
\hline $\mathrm{J} 1824+5651$ & PLCKERC030 G085.72+26.08 & 20100915 & $\begin{array}{c}20090827,20090828,20090829, \\
20100417,20100418\end{array}$ \\
\hline $\mathrm{J} 1829+4844$ & PLCKERC030 G077.20+23.49 & 20100915 & $\begin{array}{c}\text { 20090910, 20090911, 20090912, } \\
\text { 20100411, 20100412 }\end{array}$ \\
\hline $\mathrm{J} 1849+6705$ & PLCKERC030 G097.46+25.04 & $\begin{array}{c}20100723,20100803,20100915 \\
20101020,20101109\end{array}$ & $\begin{array}{c}20091012,20091013,20091014, \\
20100117,20100118,20100119, \\
20100513,20100514\end{array}$ \\
\hline $\mathrm{J} 1927+7358$ & PLCKERC030 G105.63+23.54 & 20101020 & $\begin{array}{c}20090928,20090929,20090930, \\
20100202,20100203\end{array}$ \\
\hline $\mathrm{J} 1955+5131$ & PLCKERC030 G085.28+11.76 & $20101020,20101127,20101120$ & $\begin{array}{c}\text { 20091203, 20091204, 20091205, } \\
\text { 20100506, 20100507 }\end{array}$ \\
\hline $\mathrm{J} 2005+7752$ & PLCKERC030 G110.45+22.73 & 20101020 & $\begin{array}{c}20090925,20090926,20100209, \\
20100210\end{array}$ \\
\hline $\mathrm{J} 2022+6136$ & PLCKERC030 G096.08+13.77 & $20100702,20100723,20100803$ & $\begin{array}{c}\text { 20100106, 20100107, 20100526, } \\
20100527,20100528\end{array}$ \\
\hline J2123+0535 & PLCKERC030 G058.03-30.09 & 20101109, 20091115 & 20091110, 20091111, 20100509 \\
\hline J2134-0153 & PLCKERC030 G052.38-36.49 & 20091115, 20091103 & 20091110, 20100509, 20100510 \\
\hline $\mathrm{J} 2136+0041$ & PLCKERC030 G055.47-35.57 & 20101109,20091115 & 20091112, 20100511 \\
\hline $\mathrm{J} 2139+1423$ & PLCKERC030 G068.51-27.50 & $20101127,20101120,20091115$ & 20091120, 20091121, 20100516 \\
\hline $\mathrm{J} 2148+0657$ & PLCKERC030 G063.61-34.11 & 20091115 & 20091118, 20091119, 20100516 \\
\hline $\mathrm{J} 2202+4216$ & PLCKERC030 G092.62-10.44 & 20091103 & $\begin{array}{c}20091225,20091226,20100606, \\
20100607\end{array}$ \\
\hline J2203+1725 & PLCKERC030 G075.68-29.62 & $20101127,20101120,20091103$ & $\begin{array}{c}20091201,20091202,20100524, \\
20100525\end{array}$ \\
\hline $\mathrm{J} 2203+3145$ & PLCKERC030 G085.95-18.77 & 20100702, 20091103 & $20091214,20091215,20100531$ \\
\hline J2218-0335 & PLCKERC030 G059.05-46.63 & 20091115 & $20091121,20091122,20100522$ \\
\hline J2225-0457 & PLCKERC030 G058.96-48.81 & 20091115 & 20091123, 20100523, 20100524 \\
\hline J2229-0832 & PLCKERC030 G055.15-51.70 & 20091115 & 20091122, 20100523, 20100524 \\
\hline $\mathbf{J} 2232+1143$ & PLCKERC030 G077.45-38.54 & 20091103 & 20091206, 20100601 \\
\hline $\mathrm{J} 2236+2828$ & PLCKERC030 G090.11-25.64 & 20100702,20091103 & 20091220, 20091221 \\
\hline $\mathrm{J} 2253+1608$ & PLCKERC030 G086.12-38.18 & 20100702, 20091103 & 20091215,20091216 \\
\hline J2327+0940 & PLCKERC030 G091.17-47.99 & 20100702, 20100723, 20100803 & 20091220, 20091221 \\
\hline
\end{tabular}

